\title{
A Family of Binary Univariate Nonstationary Quasi-Interpolatory Subdivision Reproducing Exponential Polynomials
}

\author{
Baoxing Zhang (D, Hongchan Zheng, Lulu Pan, Guohua Peng, and Weijie Song \\ Department of Applied Mathematics, Northwestern Polytechnical University, Xi'an, Shaanxi 710072, China \\ Correspondence should be addressed to Baoxing Zhang; baoxingzhang@yeah.net
}

Received 4 April 2019; Revised 30 June 2019; Accepted 17 October 2019; Published 4 November 2019

Academic Editor: Ibrahim Zeid

Copyright ( 92019 Baoxing Zhang et al. This is an open access article distributed under the Creative Commons Attribution License, which permits unrestricted use, distribution, and reproduction in any medium, provided the original work is properly cited.

In this paper, by suitably using the so-called push-back operation, a connection between the approximating and interpolatory subdivision, a new family of nonstationary subdivision schemes is presented. Each scheme of this family is a quasi-interpolatory scheme and reproduces a certain space of exponential polynomials. This new family of schemes unifies and extends quite a number of the existing interpolatory schemes reproducing exponential polynomials and noninterpolatory schemes like the cubic exponential B-spline scheme. For these new schemes, we investigate their convergence, smoothness, and accuracy and show that they can reach higher smoothness orders than the interpolatory schemes with the same reproduction property and better accuracy than the exponential B-spline schemes. Several examples are given to illustrate the performance of these new schemes.

\section{Introduction}

Subdivision schemes are efficient tools to generate smooth curves/surfaces from a given set of discrete control points. Over the last decades, they are shown to be important tools in many fields like CAD/CAM $[1,2]$, wavelets $[3,4]$, biomedical imaging [5], and isogeometric analysis [6]. According to whether the refinement rules depend on the recursion level, subdivision schemes can be divided into stationary and nonstationary schemes. Stationary schemes have been extensively studied, for example, in $[7,8]$. It is known that stationary schemes generate algebraic polynomials. The nonstationary schemes, however, can generate the richer function spaces, i.e., the exponential polynomial spaces and special curves/surfaces such as hyperbolas/spheres, which cannot be done using stationary schemes (see, e.g., [9-11]). Therefore, there have been continuous works on nonstationary subdivision schemes generating exponential polynomials.

In connection with the construction of such nonstationary subdivision, Romani [12] converted three exponential B-spline schemes into interpolatory schemes without changing the generation property. Conti et al. [13] transformed the nonstationary approximating schemes into interpolatory ones with the same generation property. All of these works can be seen as performed using the polynomial correction, which actually operates by taking the convex combination of the approximating subdivision masks to derive new subdivision masks, including the interpolatory ones. For other references on this method, refer [14-17] and the references therein.

Apart from polynomial correction, the push-back operation [18], a connection between the approximating and interpolatory subdivision, can also be used to derive interpolatory schemes from the approximating ones. Using this connection, Lin et al. [19] obtained interpolatory surface subdivision from the approximating subdivision. Luo and Qi [20] analyzed the interpolatory subdivision obtained by this connection systematically in the univariate case. For other references on this connection, see also [21-23] and the references therein. Yet, unlike the polynomial correction, most of the works related to the push-back operation, except the work in [24], are restricted to the stationary case, and the reproduction property of the obtained interpolatory schemes depends largely on that of the original approximating schemes [20]. In [24], the authors presented a nonstationary combined subdivision generating/reproducing different exponential polynomials using the push-back operation in a suitable way. However, when reproducing exponential polynomials, this combined scheme only reduces to the 
existing interpolatory schemes. Since interpolatory schemes are usually less smooth than the approximating ones, in this paper, we aim to give a different try on the use of the pushback operation to construct new nonstationary subdivision schemes with better properties such as reproduction of exponential polynomials with higher smoothness orders. As a by-product, this shows that, just like the polynomial correction, the push-back operation can also be used to construct a nonstationary subdivision with satisfactory properties.

In this paper, similar to [24], the displacements in the push-back operation are decomposed into small ones. Yet, to obtain the desired new nonstationary schemes, we use these small displacements in a different way. We point out that each one of these new schemes is a nonstationary quasi-interpolatory scheme. Here, a nonstationary quasi-interpolatory scheme refers to a nonstationary subdivision scheme reproducing a certain space of exponential polynomials. In fact, the nonstationary quasi-interpolatory schemes in this paper also combined approximating/interpolatory subdivision. Thus, they unify and extend quite a number of the existing interpolatory and noninterpolatory schemes reproducing exponential polynomials, including the nonstationary interpolatory 4-point scheme [25], the cubic exponential B-spline scheme. For these new schemes, we show that their asymptotical similar schemes are the schemes $S_{2 M}$ with $M \in \mathbb{Z}_{+}$in [26]. Then, based on this result, the convergence, smoothness, and accuracy can be investigated. Each one of these new schemes owns a parameter and can be seen as a parameter-dependent subdivision (see [27]). With suitable choices of this parameter, each new scheme provides a better smoothness property at the expense of slightly larger support than the interpolatory one with the same reproduction property. Besides, this parameter also provides flexibility in curve design. Moreover, each one of these new schemes owns a good approximation order due to the reproduction property. Thus, a nonstationary quasiinterpolatory scheme in this paper is also a nonstationary scheme with a good approximation order. In this way, these new schemes can own higher smoothness orders than the interpolatory ones with the same reproduction property and better accuracy than the exponential B-spline schemes. Several examples are given to illustrate the performance of these new schemes.

The rest of this paper is organized as follows: in Section 2, we recall some basic knowledge about subdivision schemes. A new family of nonstationary quasi-interpolatory schemes is constructed in Section 3, while the properties of convergence, smoothness, and accuracy are investigated in Section 4. In Section 5, we present some examples and make a comparison with the existing schemes to illustrate the performance of these new schemes. Section 6 concludes this paper.

\section{Background}

In this section, let us recall some basic definitions and results about the subdivision which form the basis of the rest of this paper. Let $l_{0}(\mathbb{Z})$ denote the linear space of real sequences with finite support. Given a sequence of initial control points $\mathbf{q}^{0}=\left\{q_{j}^{0}, j \in \mathbb{Z}\right\} \in l_{0}(\mathbb{Z})$, we consider the binary nonstationary subdivision scheme

$$
\left(\mathbf{q}^{k+1}\right)_{i}=\left(S_{\mathbf{a}^{k}} \mathbf{q}^{k}\right)_{i}:=\sum_{j} a_{i-2 j}^{k} q_{j}^{k}, \quad k \in \mathbb{N}_{0}:=\mathbb{N} \cup\{0\},
$$

where $S_{\mathrm{a}^{k}}$ is the $k$-level subdivision operator mapping $l_{0}(\mathbb{Z})$ to $l_{0}(\mathbb{Z})$, the sequence $\mathbf{a}^{k}=\left\{a_{i}^{k}, i \in \mathbb{Z}\right\} \in l_{0}(\mathbb{Z})$ is the $k$-level mask with finite support, and we denote this subdivision scheme by $\left\{S_{\mathrm{a}^{k}}\right\}_{k \geq 0}$. The $k$-level symbol corresponding to the mask $\mathbf{a}^{k}$ is $a^{k}(z)=\sum_{i \in \mathbb{Z}} a_{i}^{k} z^{i}$ with the subsymbols $a_{0}^{k}(z)=$ $\sum_{i \in \mathbb{Z}} a_{2 i}^{k} z^{2 i} \quad$ and $\quad a_{1}^{k}(z)=\sum_{i \in \mathbb{Z}} a_{2 i+1}^{k} z^{2 i+1} \quad$ satisfying $a^{k}(z)=a_{0}^{k}(z)+a_{1}^{k}(z)$.

Following [28], now we give the definition of convergence of the nonstationary subdivision.

Definition 1 (see [28]). The subdivision $\left\{S_{\mathbf{a}^{k}}\right\}_{k \geq 0}$ is termed uniformly convergent if, for an initial control sequence $\mathbf{q}^{0}$, there exists a continuous function $f_{\mathbf{q}^{0}} \in C(\mathbb{R})$ such that

$$
\lim _{k \rightarrow \infty} \max _{i \in \mathbb{Z} \cap K}\left|f_{\mathbf{q}^{0}}\left(\frac{i}{2^{k}}\right)-\left(\mathbf{q}^{k}\right)_{i}\right|=0,
$$

where $K$ is any compact set in $\mathbb{R}$ and $f_{\mathbf{q}^{0}}$ is nontrivial for at least one initial data sequence. The subdivision $\left\{S_{\mathrm{a}^{k}}\right\}_{k \geq 0}$ is termed $C^{l}$ convergent if the limit function $f_{\mathbf{q}^{0}}$ has continuous derivatives up to order $l$ with $l \in \mathbb{Z}_{+}$.

The following definitions and results are needed to investigate the convergence and smoothness of nonstationary subdivision schemes.

Definition 2 (see [29]). A nonstationary subdivision scheme $\left\{S_{\mathrm{a}^{k}}\right\}_{k \geq 0}$ is said to be asymptotic similar to the stationary subdivision scheme $S_{\mathbf{a}}$ if the masks $\left\{\mathbf{a}^{k}\right\}_{k \geq 0}$ and $\{\mathbf{a}\}$ have the same support $\mathcal{U}$ (i.e., $a_{i}^{k}=a_{i}=0$ for $i \notin \mathscr{U}$ ) and satisfy

$$
\lim _{k \rightarrow \infty} a_{i}^{k}=a_{i}, \quad i \in \mathcal{U} .
$$

Definition 3 (see [30]). Let $D^{n}$ be the $n$-th order differential operator. A binary nonstationary subdivision scheme $\left\{S_{\mathbf{a}^{k}}\right\}_{k \geq 0}$ is said to satisfy approximate sum rules of order $r+1, r \in \mathbb{N}_{0}$, if

$$
\begin{aligned}
\mu^{k} & :=\left|a^{k}(1)-2\right|, \\
\delta^{k} & :=\max _{0 \leq \eta \leq r}\left|2^{-k \eta} D^{\eta} a^{k}(-1)\right|,
\end{aligned}
$$

satisfying

$$
\begin{aligned}
\sum_{k} \mu^{k}<\infty, \\
\sum_{k} 2^{k r} \delta^{k}<\infty .
\end{aligned}
$$

Theorem 1 (see [30]). Assume that the nonstationary subdivision scheme $\left\{S_{\mathrm{a}^{k}}\right\}_{k \geq 0}$ satisfies approximate sum rules of order $r+1, r \in \mathbb{N}_{0}$ and is asymptotic similar to a $C^{r}$-convergent stationary scheme $S_{\mathrm{a}}$. Then, the nonstationary scheme $\left\{S_{\mathbf{a}^{k}}\right\}_{k \geq 0}$ is also $C^{r}$ convergent.

Apart from convergence and smoothness, the property of exponential polynomial generation and reproduction (see, for example, [31] for their details) is also important for 
subdivision schemes due to the close relationship with approximation order and the use in modeling objects of different shapes. Thus, we now review the definition of exponential polynomial spaces and related results as follows:

Definition 4 (see [31]). Let $T \in \mathbb{Z}_{+}$, and let $\gamma=\left\{\gamma_{0}, \gamma_{1}\right.$, $\left.\ldots, \gamma_{T}\right\}$ with $\gamma_{T} \neq 0$ be a finite set of real or imaginary numbers. The space of exponential polynomials $V_{T, \gamma}$ is defined as

$$
V_{T, \mathcal{Y}}:=\left\{f: \mathbb{R} \longrightarrow \mathbb{C}, f \in C^{T}(\mathbb{R}): \sum_{j=0}^{T} \gamma_{j} D^{j} f=0\right\} .
$$

The exponential polynomial space $V_{T, \gamma}$ can be characterized by the following lemma:

Lemma 1 (see [31]). Let $\gamma(z)=\sum_{j=0}^{T} \gamma_{j} z^{j}$ and denote by $\left\{\left(\theta_{i}, \tau_{i}\right)\right\}_{i=0, \ldots, N}$ the set of zeros with multiplicity, satisfying

$$
D^{r} \gamma\left(\theta_{i}\right)=0, \quad r=0, \ldots, \tau_{i}-1, i=0, \ldots, N .
$$

Then,

$$
\begin{aligned}
T & =\sum_{i=0}^{N} \tau_{i}, \\
V_{T, \gamma} & :=\operatorname{span}\left\{x^{r} e^{\theta_{i} x}, \quad r=0, \ldots, \tau_{i}-1, i=0, \ldots, N\right\} .
\end{aligned}
$$

The following two theorems, which can be deduced from Proposition 4.2 and Theorem 4.4 in [32], will be useful in discussing the generation/reproduction property of nonstationary subdivision schemes.

Theorem 2. A nonstationary binary subdivision scheme associated with symbols $\left\{a^{k}(z)\right\}_{k \geq 0}$ generates $V_{T, \gamma}$ if

$$
\begin{aligned}
D^{r} a^{k}\left(-z_{i}^{k}\right)= & 0, \quad r=0, \ldots, \tau_{i}-1, \\
& \text { for } z_{i}^{k}:=e^{-\theta_{i} / 2^{k+1}}, i=1, \ldots, N .
\end{aligned}
$$

Theorem 3. With $z_{i}^{k}=e^{\theta_{i} / 2^{k+1}}, i=1, \ldots, N$, a binary nonstationary subdivision scheme associated with symbols $\left\{a^{k}(z)\right\}_{k \geq 0}$ reproduces $V_{T, \gamma}$ if it generates $V_{T, \gamma}$ and there exists a shift parameter $p$ such that for each $k \geq 0$

$$
\begin{aligned}
D^{r} a^{k}\left(z_{i}^{k}\right) & =2\left(z_{i}^{k}\right)^{p-r} q_{r}(p), \\
q_{r}(p) & = \begin{cases}\prod_{j=0}^{r-1}(p-j), & r=1, \ldots, \tau_{i}-1, \\
1, & r=0 .\end{cases}
\end{aligned}
$$

\section{Nonstationary Quasi-Interpolatory Schemes Reproducing Exponential Polynomials}

This section is devoted to the construction of the new family of nonstationary subdivision schemes reproducing exponential polynomials. Before that, we first give an exponential polynomial space, which specializes the space in Lemma 1 and generalizes the space in ([24], Section 6).

Given $n, l \in \mathbb{Z}_{+}$, let the set of zeros with multiplicity in Lemma 1 be given as

$$
\begin{aligned}
\Gamma \Lambda_{n}:= & \left\{\left(0,2 \tau_{0}\right),\left( \pm j t, \tau_{j}\right): j=1, \ldots, l, \tau_{0} \leq 2, \tau_{0}, \tau_{1} \geq 1,\right. \\
& \left.\tau_{0}+\sum_{j=1}^{l} \tau_{j}=n+1\right\},
\end{aligned}
$$

where $t \in \mathbb{R}^{+} \cup \iota[0, \pi)$ and $\iota^{2}=-1$. In other words, we assume $\gamma(z)$ in Lemma 1 has $N=2 l+1$ pairwise distinct zeros, and its total number of zeros is $T=2 \sum_{j=0}^{l} \tau_{j}=2 n+2$. Then, the exponential polynomial space in Lemma 1 , which is actually a $2 n+2$ dimensional space, can now be rewritten as

$$
\begin{aligned}
\operatorname{EP}_{\Gamma \Lambda_{n}}:= & \operatorname{span}\left\{1, x, \ldots, x^{2 \tau_{0}-1}\right\} \cup \operatorname{span}\left\{x^{r} e^{ \pm j \mathrm{tx}}: 0 \leq r \leq \tau_{j}-1,\right. \\
& \left.\cdot\left( \pm j t, \tau_{j}\right) \in \Gamma \Lambda_{n}, j=1, \ldots, l\right\} .
\end{aligned}
$$

Here, when $n=0$, let $\Gamma \Lambda_{0}:=\{(0,2)\}$ and we get $\mathrm{EP}_{\Gamma \Lambda_{0}}:=\operatorname{span}\{1, x\}$. When $n=1$, we have $\tau_{0}+\sum_{j=1}^{l} \tau_{j}=2$. Since $\tau_{0}, \tau_{1} \geq 1$, we have $l=1, \quad \tau_{0}=\tau_{1}=1$ and then $\Gamma \Lambda_{1}=\{(0,2),( \pm t, 1)\}$. Thus, $\mathrm{EP}_{\Gamma \Lambda_{1}}=\operatorname{span}\left\{1, x, e^{ \pm \mathrm{tx}}\right\}$. Note that compared with the space in ([24] Section 6), this new one contains more exponentials like $x^{r} e^{ \pm \mathrm{tx}}, r \geq 2$.

Now, we try to construct the desired nonstationary subdivision schemes, which are, in fact, quasi-interpolatory ones reproducing exponential polynomials in $\mathrm{EP}_{\Gamma \Lambda_{n}}$. In order to describe the construction clearly, we first derive the schemes reproducing $\mathrm{EP}_{\Gamma \Lambda_{0}}$ and $\mathrm{EP}_{\Gamma \Lambda_{1}}$. Then, by taking a generalization, we obtain the general scheme reproducing $\mathrm{EP}_{\Gamma \Lambda_{n}}$.

3.1. The Nonstationary Quasi-Interpolatory Schemes Reproducing $E P_{\Gamma \Lambda_{0}}$ and $E P_{\Gamma \Lambda_{1}}$. Now, we construct the desired nonstationary schemes reproducing $\mathrm{EP}_{\Gamma \Lambda_{0}}$ and $\mathrm{EP}_{\Gamma \Lambda_{1}}$.

3.1.1. The Cubic Exponential B-Spline Scheme and the Nonstationary Quasi-Interpolatory Scheme Reproducing $E P_{\Gamma \Lambda_{0}}$. We start from the cubic exponential B-spline scheme. As it is known, for $k \geq 0$, the cubic exponential Bspline scheme generates the refined data sequence $\left\{P_{i}^{k+1}\right\}_{i \in \mathbb{Z}}$ from the coarser data sequence $\left\{p_{i}^{k}\right\}_{i \in \mathbb{Z}}$ through the refinement rules

$$
\left\{\begin{array}{l}
P_{2 i}^{k+1}=\frac{1}{4\left(1+v^{k+1}\right)}\left(P_{i-1}^{k}+P_{i+1}^{k}\right)+\frac{1+2 v^{k+1}}{2\left(1+v^{k+1}\right)} p_{i}^{k}, \\
P_{2 i+1}^{k+1}=\frac{1}{2}\left(p_{i}^{k}+P_{i+1}^{k}\right),
\end{array}\right.
$$


where

$$
\begin{aligned}
v^{k+1} & :=\frac{e^{t_{k+1}}+e^{-t_{k+1}}}{2}, \\
t_{k+1} & =\frac{t}{2^{k+1}},
\end{aligned}
$$

$$
t \in \mathbb{R}^{+} \cup \iota[0, \pi) .
$$

The cubic exponential B-spline scheme (1) is known to generate the space $\mathrm{EP}_{\Gamma \Lambda}$. Based on this scheme, by making a suitable modification to its refinement rules [24], a nonstationary combined subdivision scheme can be obtained as

$$
\left\{\begin{array}{l}
P_{2 i}^{k+1}=\widetilde{P}_{2 i}^{k+1}+\alpha_{0}^{k} \Delta p_{i}^{k}, \\
P_{2 i+1}^{k+1}=\widetilde{P}_{2 i+1}^{k+1}-\beta_{0}^{k}\left(\Delta p_{i}^{k}+\Delta P_{i+1}^{k}\right),
\end{array}\right.
$$

where $\alpha_{0}^{k}$ and $\beta_{0}^{k}$ are constants depending on $k, \Delta p_{i}^{k}:=$ $-\left(P_{i-1}^{k}-2 p_{i}^{k}+P_{i+1}^{k}\right)$ and $\widetilde{P}_{2 i}^{k+1}=\left(1 /\left(4\left(1+v^{k+1}\right)\right)\right)\left(P_{i-1}^{k}+\right.$ $\left.P_{i+1}^{k}\right)+\left(\left(1+2 v^{k+1}\right) /\left(2\left(1+v^{k+1}\right)\right)\right) p_{i}^{k}, \quad \widetilde{P}_{2 i+1}^{k+1}=(1 / 2)\left(p_{i}^{k}+\right.$ $\left.P_{i+1}^{k}\right)$ are the two points provided by the cubic exponential B-spline scheme (1).

The nonstationary combined scheme (3) can be seen as obtained by moving the points $\widetilde{P}_{2 i}^{k+1}$ and $\widetilde{P}_{2 i+1}^{k+1}$ to new positions according to the displacements $\alpha_{0}^{k} \Delta p_{i}^{k}$ and $-\beta_{0}^{k}\left(\Delta p_{i}^{k}+\Delta P_{i+1}^{k}\right)$, respectively. From [24], scheme (3) unifies several existing subdivision schemes, including the nonstationary interpolatory 4-point scheme [25] and the cubic exponential B-spline scheme (1). In particular, when $\beta_{0}^{k}=0$, the combined scheme (3) can be rewritten as

$$
\left\{\begin{array}{l}
P_{2 i}^{k+1}=\left(\frac{1}{4\left(1+v^{k+1}\right)}-\alpha_{0}^{k}\right)\left(P_{i-1}^{k}+P_{i+1}^{k}\right) \\
\quad+\left(\frac{1+2 v^{k+1}}{2\left(1+v^{k+1}\right)}+2 \alpha_{0}^{k}\right) p_{i}^{k}, \\
P_{2 i+1}^{k+1}=\frac{1}{2}\left(p_{i}^{k}+P_{i+1}^{k}\right) .
\end{array}\right.
$$

By writing down the symbols of the scheme (4), from Theorem 3, it can be seen that the scheme (4), if convergent, reproduces $\mathrm{EP}_{\Gamma \Lambda_{0}}$. Besides, when $\alpha_{0}^{k}=0$, the scheme (4) reduces to the cubic exponential B-spline scheme (1), and when $\alpha_{0}^{k}=1 /\left(4\left(1+v^{k+1}\right)\right)$, it becomes the D-D 2-point scheme. In this way, the scheme (4) is a nonstationary scheme reproducing $\mathrm{EP}_{\Gamma \Lambda_{0}}$, which combines the D-D 2point scheme and the cubic exponential B-spline scheme (1).

3.1.2. The Nonstationary Quasi-Interpolatory Scheme Reproducing $E P_{\Gamma \Lambda}$. Now, we try to obtain the quasi-interpolatory subdivision reproducing $\mathrm{EP}_{\Gamma \Lambda_{1}}$, which reproduces more exponential polynomials than the scheme (4).

In fact, we modify both rules of the scheme (4) by adding the term $-\beta_{0}^{k}\left(\Delta p_{i}^{k}+\Delta P_{i+1}^{k}\right)$ to the second rule of (4) and adding additionally the term $\alpha_{1}^{k}\left(\Delta P_{i-1}^{k}+\Delta P_{i+1}^{k}\right)$ to its first rule to obtain the following new scheme:

$$
\left\{\begin{array}{l}
P_{2 i}^{k+1}=\widetilde{P}_{2 i}^{k}+\alpha_{0}^{k} \Delta p_{i}^{k}+\alpha_{1}^{k}\left(\Delta P_{i-1}^{k}+\Delta P_{i+1}^{k}\right), \\
P_{2 i+1}^{k+1}=\widetilde{P}_{2 i+1}^{k}-\beta_{0}^{k}\left(\Delta p_{i}^{k}+\Delta P_{i+1}^{k}\right) .
\end{array}\right.
$$

The scheme (5) can be seen as obtained by moving the points $\widetilde{P}_{2 i}^{k+1}$ and $\widetilde{P}_{2 i+1}^{k+1}$, generated by the cubic exponential Bspline scheme (1), according to the displacements $\alpha_{0}^{k} \Delta p_{i}^{k}+$ $\alpha_{1}^{k}\left(\Delta P_{i-1}^{k}+\Delta P_{i+1}^{k}\right)$ and $-\beta_{0}^{k}\left(\Delta p_{i}^{k}+\Delta P_{i+1}^{k}\right)$, respectively. In this way, the corresponding $k$-level symbol can be written as

$$
a^{k}(z)=a_{0}^{k}(z)+a_{1}^{k}(z),
$$

where

$$
\left\{\begin{array}{c}
a_{0}^{k}(z)=-\alpha_{1}^{k}\left(z^{4}+z^{-4}\right)+\left(\frac{1}{4\left(1+v^{k+1}\right)}-\alpha_{0}^{k}+2 \alpha_{1}^{k}\right) \\
\cdot\left(z^{2}+z^{-2}\right)+\left(\frac{2 v^{k+1}+1}{2\left(1+v^{k+1}\right)}+2 \alpha_{0}^{k}-2 \alpha_{1}^{k}\right) \\
a_{1}^{k}(z)=\beta_{0}^{k}\left(z^{3}+z^{-3}\right)+\left(\frac{1}{2}-\beta_{0}^{k}\right)\left(z+z^{-1}\right) .
\end{array}\right.
$$

For the scheme (5), from Theorem 3, by solving the linear system,

$$
\begin{aligned}
D^{i} a^{k}(-1) & =0, \\
D^{i} a^{k}(1) & =2 \delta_{i, 0}, \\
a^{k}\left(-e^{ \pm t_{k+1}}\right) & =0, \\
a^{k}\left(e^{ \pm t_{k+1}}\right) & =2, \\
i & =0,1 .
\end{aligned}
$$

We can find its solution containing a free parameter, which we denote by $\omega^{k}$. Here, we set $\omega^{k}:=\alpha_{1}^{k}$. Then, with the parameters in this solution, the scheme (5), if convergent, reproduces $\mathrm{EP}_{\Gamma \Lambda_{1}}$. We denote this new scheme reproducing $\mathrm{EP}_{\Gamma \Lambda_{1}}$ by $\left\{S_{\mathrm{a}_{\Gamma \Lambda_{1}, \omega^{k}}}\right\}_{k \geq 0}$, and the corresponding $k$-level symbol can be written as

$$
\begin{aligned}
a_{\Gamma \Lambda_{1}, \omega^{k}}^{k}(z)= & -\omega^{k}\left(z^{4}+z^{-4}\right)-\frac{1}{8 v^{k+1}\left(1+v^{k+1}\right)} \\
& \cdot\left(z^{3}+z^{-3}\right)+4 \omega^{k}\left(v^{k+1}\right)^{2}\left(z^{2}+z^{-2}\right) \\
& +\frac{\left(1+2 v^{k+1}\right)^{2}}{8 v^{k+1}\left(1+v^{k+1}\right)}\left(z+z^{-1}\right)+1 \\
& +2 \omega^{k}\left(1-4\left(v^{k+1}\right)^{2}\right) .
\end{aligned}
$$

In addition, Theorem 2 implies that the scheme $\left\{S_{\mathbf{a}^{k} \Lambda_{1}, \omega^{k}}\right\}_{k \geq 0}$ also generates the space 


$$
\mathrm{EP}_{\Gamma \Lambda_{2}}:=\left\{1, x, x^{2}, x^{3}, e^{ \pm t x}\right\} \text { with } \Gamma \Lambda_{2}=\{(0,4),( \pm t, 1)\},
$$

when $\omega^{k}=\left(v^{k+1}+2\right) /\left(32 v^{k+1}\left(1+v^{k+1}\right)^{2}\right)$. Note that, when $\omega^{k}=0$, the scheme $\left\{S_{\mathfrak{a}_{\Gamma \Lambda_{1}, \omega^{k}}^{k}}\right\}_{k \geq 0}$ reduces to the nonstationary interpolatory 4-point scheme [25], and when $\omega^{k}=\left(v^{k+1}+2\right) /\left(32 v^{k+1}\left(1+v^{k+1}\right)^{2}\right)$, it actually becomes the exponential pseudospline scheme generating $\mathrm{EP}_{\Gamma \Lambda_{2}}$ (22) and reproducing $\mathrm{EP}_{\Gamma \Lambda_{1}}$ [33]. Here, we denote by $e_{\Gamma \Lambda_{1}, 1}^{k}(z)$ and $e_{\Gamma \Lambda_{1}, 2}^{k}(z)$ the $k$-level symbols of the nonstationary interpolatory 4-point scheme [25] and the aforementioned exponential pseudospline scheme, which can be written as

$$
\left\{\begin{array}{l}
e_{\Gamma \Lambda_{1}, 1}^{k}(z)=-\frac{1}{8 v^{k+1}\left(1+v^{k+1}\right)}\left(z^{3}+z^{-3}\right) \\
+\frac{\left(1+2 v^{k+1}\right)^{2}}{8 v^{k+1}\left(1+v^{k+1}\right)}\left(z+z^{-1}\right)+1, \\
e_{\Gamma \Lambda_{1}, 2}^{k}(z)=\frac{(1+z)^{4}}{8} \frac{z^{2}+2 v^{k+1} z+1}{2\left(1+v^{k+1}\right)} \\
\cdot\left(-\frac{v^{k+1}+2}{2 v^{k+1}\left(v^{k+1}+1\right)}\left(1+z^{2}\right)+\frac{\left(v^{k+1}\right)^{2}+2 v^{k+1}+2}{v^{k+1}\left(1+v^{k+1}\right)} z\right) z^{-4},
\end{array}\right.
$$

respectively. Now, let $\theta^{k}=\left(\left(32 v^{k+1}\left(1+v^{k+1}\right)^{2}\right) /\left(v^{k+1}+\right.\right.$ 2)) $\omega^{k}$, and then the $k$-level symbol $a_{\Gamma \Lambda_{1}, \omega^{k}}^{k}(z)$ can be rewritten as

$$
a_{\Gamma \Lambda_{1}, \omega^{k}}^{k}(z)=\left(1-\theta^{k}\right) e_{\Gamma \Lambda_{1}, 1}^{k}(z)+\theta^{k} e_{\Gamma \Lambda_{1}, 2}^{k}(z) .
$$

In this way, we obtain a new nonstationary scheme reproducing $\mathrm{EP}_{\Gamma \Lambda_{1}}$, which combines the nonstationary interpolatory 4-point scheme as shown in [25] and the noninterpolatory one with the $k$-level symbol $e_{\Gamma \Lambda_{1}, 2}^{k}(z)$. Note that the $k$-level symbol in (24) is actually a convex combination of the symbols $e_{\Gamma \Lambda_{1}, 1}^{k}(z)$ and $e_{\Gamma \Lambda_{1}, 2}^{k}(z)$ for $0 \leq \theta^{k} \leq 1$.

Figure 1 shows the basic limit functions of this new scheme reproducing $\mathrm{EP}_{\Gamma \Lambda_{1}}$ with $v^{0}=\cos (\pi / 4)$ and $\omega^{k}=-0.015,0,0.015,0.03$. Figure 2 shows the curves generated by this new scheme reproducing $\mathrm{EP}_{\Gamma \Lambda_{1}}$ with different values of $\omega^{k}$ and $v^{0}$. In particular, when $v^{0}=\cos (\pi / 4)$ (solid line in the left column), 1 (solid line in the middle column), and $\cosh (3 / 5)$ (solid line in the right column), Figure 2 shows the reproduction of the circle, parabola, and hyperbola, respectively. From Figures 1 and 2, we can also see the effect of the parameter $\omega^{k}$ on the shape of the limit functions of this new scheme.

3.2. Nonstationary Quasi-Interpolatory Schemes Reproducing $E P_{\Gamma \Lambda_{n}}$. As it can be seen from Section 3.1, the two newly derived schemes reproducing $\mathrm{EP}_{\Gamma \Lambda_{0}}$ and $\mathrm{EP}_{\Gamma \Lambda_{1}}$ are actually obtained by suitably using the push-back operation. Now, we follow this method and take a generalization to derive nonstationary schemes reproducing general exponential polynomials, which are in $\mathrm{EP}_{\Gamma \Lambda_{n}}$.

Specifically speaking, we use more terms like $\alpha_{1}^{k}\left(\Delta P_{i-1}^{k}+\right.$ $\left.\Delta P_{i+1}^{k}\right)$ and $\beta_{0}^{k}\left(\Delta p_{i}^{k}+\Delta P_{i+1}^{k}\right)$ to modify the first and second rules of the scheme (4) to get

$$
\left\{\begin{array}{l}
P_{2 i}^{k+1}=\widetilde{P}_{2 i}^{k+1}+\alpha_{0}^{k} \Delta p_{i}^{k}+\Delta_{2 i, n}^{k} P^{k}, \\
P_{2 i+1}^{k+1}=\widetilde{P}_{2 i+1}^{k+1}-\Delta_{2 i+1, n}^{k} P^{k},
\end{array}\right.
$$

where

$$
\begin{array}{r}
\Delta_{2 i, n}^{k} P^{k}=\sum_{j=1}^{N} \alpha_{j}^{k}\left(\Delta P_{i-j}^{k}+\Delta P_{i+j}^{k}\right), \\
\Delta_{2 i+1, n}^{k} P^{k}=\sum_{j=0}^{n-1} \beta_{j}^{k}\left(\Delta P_{i-j}^{k}+\Delta P_{i+1+j}^{k}\right), \\
n \geq 0,
\end{array}
$$

with the $k$-level symbol

$$
a^{k}(z)=a_{0}^{k}(z)+a_{1}^{k}(z),
$$

where

$$
\left\{\begin{array}{l}
a_{0}^{k}(z)=\frac{1+2 v^{k+1}}{2\left(1+v^{k+1}\right)}+2 \alpha_{0}^{k}-2 \alpha_{1}^{k}+\frac{1}{4\left(1+v^{k+1}\right)} \\
\cdot\left(z^{2}+z^{-2}\right)+\sum_{j=1}^{n-1}\left(-\alpha_{j-1}^{k}+2 \alpha_{j}^{k}-\alpha_{j+1}^{k}\right) \\
\cdot\left(z^{2 j}+z^{-2 j}\right)+\left(2 \alpha_{n}^{k}-\alpha_{n-1}^{k}\right)\left(z^{2 n}+z^{-2 n}\right) \\
-\alpha_{n}^{k}\left(z^{2 n+2}+z^{-2 n-2}\right), \\
a_{1}^{k}(z)=\left(\frac{1}{2}-\beta_{0}^{k}+\beta_{1}^{k}\right)\left(z+z^{-1}\right) \\
+\sum_{j=1}^{n-2}\left(\beta_{j-1}^{k}-2 \beta_{j}^{k}+\beta_{j+1}^{k}\right)\left(z^{2 j+1}+z^{-2 j-1}\right) \\
+\left(\beta_{n-2}^{k}-2 \beta_{n-1}^{k}\right)\left(z^{2 n-1}+z^{-2 n+1}\right)+\beta_{n-1}^{k}\left(z^{2 n+1}+z^{-2 n-1}\right) .
\end{array}\right.
$$

In other words, the scheme (10) is obtained by moving the points $\widetilde{P}_{2 i}^{k+1}$ and $\widetilde{P}_{2 i+1}^{k+1}$, generated by the cubic exponential B-spline scheme (1), to new positions according to the displacements $\alpha_{0}^{k} \Delta p_{i}^{k}+\Delta_{2 i, n}^{k}$ and $-\Delta_{2 i+1, n}^{k}$, respectively. In particular, when $n=0$, we have $\Delta_{2 i, 0}^{k}=\Delta_{2 i+1,0}^{k}=0$ and the scheme (10) reduces to the scheme (4). Besides, when $\alpha_{1}^{k}=\cdots=\alpha_{n}^{k}=0$, the scheme (10) becomes the generalized combined scheme ([24], formula (25)).

Now, we focus on the reproduction/generation property of the scheme (10) and show that by suitably choosing the parameters, the desired scheme reproducing $\mathrm{EP}_{\Gamma \Lambda_{n}}$ can be obtained. In fact, we have the following result. 


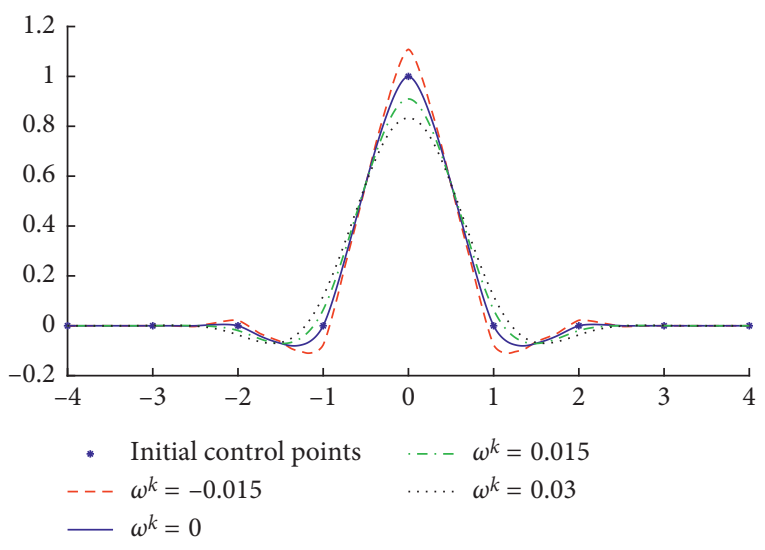

Figure 1: Basic limit functions of the newly obtained scheme reproducing $\mathrm{EP}_{\Gamma \Lambda_{1}}$ with $\omega^{k}=-0.015,0,0.015,0.03$ and $v^{0}=\cos$ $(\pi / 4)$.

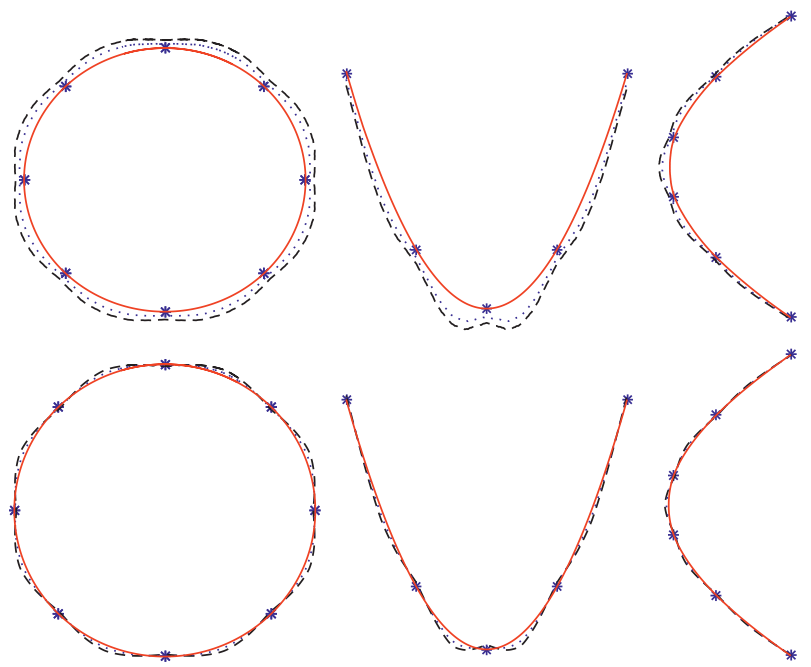

Figure 2: Curves generated by the newly obtained scheme reproducing $\mathrm{EP}_{\Gamma \Lambda_{1}}$ with $\omega^{k}=0.14$ (top row) and -0.01 (bottom row); $v^{0}=0$ (dashed line), 0.3 (dotted line), and $\cos (\pi / 4)$ (solid line in the left column); $v^{0}=1$ (solid line in the middle column); and $v^{0}=\cosh (3 / 5)$ (solid line in the right column).

Theorem 4. Given the set $\Gamma \Lambda_{n}$ with $n \geq 1$, with suitable choices of the parameters $\alpha_{0}^{k}, \ldots, \alpha_{n}^{k}, \beta_{0}^{k}, \ldots, \beta_{n-1}^{k}$, the scheme (10), if convergent, reproduces $\mathrm{EP}_{\Gamma \Lambda_{n}}$.

Proof. Since the case of $n=1$ has been investigated in Section 3.1 .2 , here we only need to investigate the case of $n \geq 2$. From Theorem 3, the scheme (10) reproduces $\mathrm{EP}_{\Gamma \Lambda_{n}}$, if for $p=0,1, \ldots, 2 \tau_{0}-1$ and $q=0,1, \ldots, \tau_{j}-1, j=1, \ldots, l$, the corresponding $k$-level symbol in (27) satisfies

$$
\begin{aligned}
D^{p} a^{k}(-1) & =0, \\
D^{q} a^{k}\left(-e^{ \pm j t_{k+1}}\right) & =0, \\
D^{p} a^{k}(1) & =2 \delta_{p, 0}, \\
D^{q} a^{k}\left(e^{ \pm j t_{k+1}}\right) & =2 \delta_{q, 0} .
\end{aligned}
$$

The two subsymbols $a_{0}^{k}(z)$ and $a_{1}^{k}(z)$ satisying $a^{k}(z)=$ $a_{0}^{k}(z)+a_{1}^{k}(z)$ satisfy

$$
\begin{aligned}
a_{0}^{k}(z) & =a_{0}^{k}(-z), \\
a_{1}^{k}(-z) & =-a_{1}^{k}(z),
\end{aligned}
$$

respectively. Thus, for $r \in \mathbb{N}_{0}, D^{r} a_{0}^{k}(z)$ is an even function if $r$ is an even number and odd function if $r$ is an odd number, while $D^{r} a_{1}^{k}(z)$ is an even function if $r$ is an odd number and odd function if $r$ is an even number. Therefore, for $r \in \mathbb{N}_{0}$, the subsymbols $a_{0}^{k}(z)$ and $a_{1}^{k}(z)$ satisfy

$$
\begin{aligned}
& D^{r} a_{0}^{k}\left(-e^{ \pm v t_{k+1}}\right)=(-1)^{r} D^{r} a_{0}^{k}\left(e^{ \pm \nu t_{k+1}}\right), \\
& D^{r} a_{1}^{k}\left(-e^{ \pm v t_{k+1}}\right)=(-1)^{r+1} D^{r} a_{1}^{k}\left(e^{ \pm \nu t_{k+1}}\right),
\end{aligned}
$$

where $t_{k+1}$ is defined as in (14). In this way, from (29), for $p=0,1, \ldots, 2 \tau_{0}-1$, we have

$$
\left\{\begin{array}{l}
D^{p} a^{k}(-1)=D^{p} a_{0}^{k}(-1)+D^{p} a_{1}^{k}(-1)=(-1)^{p} D^{p} a_{0}^{k}(1) \\
\quad+(-1)^{p+1} D^{p} a_{1}^{k}(1)=0, \\
D^{p} a^{k}(1)=D^{p} a_{0}^{k}(1)+D^{p} a_{1}^{k}(1)=2 \delta_{p, 0} .
\end{array}\right.
$$

This leads to the result that $D^{p} a_{0}^{k}(1)=D^{p} a_{1}^{k}(1)=\delta_{p, 0}$. The other two equations in (29) can be dealt with in a similar way. In this way, the linear system (29) is actually equivalent to the following two ones:

$$
\begin{aligned}
& \left\{\begin{array}{l}
D^{p} a_{0}^{k}(1)=\delta_{p, 0}, \\
D^{q} a_{0}^{k}\left(e^{ \pm j t_{k+1}}\right)=\delta_{q, 0},
\end{array}\right. \\
& \left\{\begin{array} { l } 
{ D ^ { p } a _ { 1 } ^ { k } ( 1 ) = \delta _ { p , 0 } , } \\
{ D ^ { q } a _ { 1 } ^ { k } ( e ^ { \pm j t _ { k + 1 } } ) = \delta _ { q , 0 } , }
\end{array} \text { with } \left\{\begin{array}{l}
p=0,1, \ldots, 2 \tau_{0}-1, \\
q=0,1, \ldots, \tau_{j}-1, j=1, \ldots, l .
\end{array}\right.\right.
\end{aligned}
$$

Now, let us find the desired $\alpha_{0}^{k}, \ldots, \alpha_{n}^{k}, \beta_{0}^{k}, \ldots, \beta_{n-1}^{k}$ by solving the linear systems in (33). For the first linear system in (33), we make a substitution as follows:

$$
\begin{aligned}
h_{0}^{k} & =2\left(\frac{1+2 v^{k+1}}{4\left(1+v^{k+1}\right)}+\alpha_{0}^{k}-\alpha_{1}^{k}\right), \\
h_{1}^{k} & =\frac{1}{4\left(1+v^{k+1}\right)}-\alpha_{0}^{k}+2 \alpha_{1}^{k}-\alpha_{2}^{k}, \\
h_{j}^{k} & =-\alpha_{j-1}^{k}+2 \alpha_{j}^{k}-\alpha_{j+1}^{k}(j=2, \ldots, n-1), \ldots, \\
h_{n}^{k} & =2 \alpha_{n}^{k}-\alpha_{n-1}^{k}, \\
h_{n+1}^{k} & =-\alpha_{n}^{k} .
\end{aligned}
$$

Then, the first linear system in (33) can be rewritten as

$$
\mathbf{A}^{k} \mathbf{h}^{k}=\mathbf{g}
$$

Here, $\mathbf{A}^{k}$ is the $(2 n+2) \times(n+2)$ matrix $\mathbf{A}^{k}=\left(\left(\mathbf{A}_{0}^{k}\right)^{\top}\right.$, $\left.\left(\mathbf{A}_{1}^{k}\right)^{\top},\left(\bar{A}_{1}^{k}\right)^{\top}, \ldots,\left(\mathbf{A}_{l}^{k}\right)^{\top},\left(\bar{A}_{l}^{k}\right)^{\top}\right)^{\top}$ with 


$$
\begin{aligned}
& \mathbf{A}_{0}^{k}=\left(\begin{array}{cccc}
1 & D^{0}\left(z^{2}+z^{-2}\right)(1) & \ldots & D^{0}\left(z^{2(n+1)}+z^{-2(n+1)}\right)(1) \\
0 & D^{1}\left(z^{2}+z^{-2}\right)(1) & \ldots & D^{1}\left(z^{2(n+1)}+z^{-2(n+1)}\right)(1) \\
\vdots & \vdots & \vdots & \vdots \\
0 & D^{2 \tau_{0}-1}\left(z^{2}+z^{-2}\right)(1) & \ldots & D^{2 \tau_{0}-1}\left(z^{2(n+1)}+z^{-2(n+1)}\right)(1)
\end{array}\right), \\
& \mathbf{A}_{j}^{k}=\left(\begin{array}{cccc}
1 & D^{0}\left(z^{2}+z^{-2}\right)\left(e^{j t_{k+1}}\right) & \ldots & D^{0}\left(z^{2(n+1)}+z^{-2(n+1)}\right)\left(e^{j t_{k+1}}\right) \\
0 & D^{1}\left(z^{2}+z^{-2}\right)\left(e^{j t_{k+1}}\right) & \ldots & D^{1}\left(z^{2(n+1)}+z^{-2(n+1)}\right)\left(e^{j t_{k+1}}\right) \\
\vdots & \vdots & \vdots & \vdots \\
0 & D^{\tau_{j}-1}\left(z^{2}+z^{-2}\right)\left(e^{j t_{k+1}}\right) & \ldots & D^{\tau_{j}-1}\left(z^{2(n+1)}+z^{-2(n+1)}\right)\left(e^{j t_{k+1}}\right)
\end{array}\right), \quad j=1, \ldots, l, \\
& \mathbf{A}_{j}^{k}=\left(\begin{array}{cccc}
1 & D^{0}\left(z^{2}+z^{-2}\right)\left(e^{-j t_{k+1}}\right) & \ldots & D^{0}\left(z^{2(n+1)}+z^{-2(n+1)}\right)\left(e^{-j t_{k+1}}\right) \\
0 & D^{1}\left(z^{2}+z^{-2}\right)\left(e^{-j t_{k+1}}\right) & \ldots & D^{1}\left(z^{2(n+1)}+z^{-2(n+1)}\right)\left(e^{-j t_{k+1}}\right) \\
\vdots & \vdots & \vdots & \vdots \\
0 & D^{\tau_{j}-1}\left(z^{2}+z^{-2}\right)\left(e^{-j t_{k+1}}\right) & \ldots & D^{\tau_{j}-1}\left(z^{2(n+1)}+z^{-2(n+1)}\right)\left(e^{-j t_{k+1}}\right)
\end{array}\right), \quad j=1, \ldots, l,
\end{aligned}
$$

where $\mathbf{h}^{k}=\left(h_{0}^{k}, \ldots, h_{n+1}^{k}\right)^{\top}$ and $\mathbf{g}$ is the $(2 n+2) \times 1$ vector $\mathbf{g}=$ $\left(2 \tau_{0}-1\right)$-times $\quad\left(\tau_{1}-1\right)$-times $\quad\left(\tau_{1}-1\right)$-times $\quad\left(\tau_{l}-1\right)$-times $(1, \overbrace{0, \ldots, 0}, 1, \overbrace{0, \ldots, 0}, 1, \overbrace{0, \ldots, 0}, \ldots, 1, \overbrace{0, \ldots, 0}, 1$, $\left(\tau_{l}-1\right)$-times

$\overbrace{0, \ldots, 0})$. Note that with the substitution in (34), $a_{0}^{k}(z)$ satisfies

$$
\begin{aligned}
& D^{1} a_{0}^{k}(1)=0, \\
& D^{3} a_{0}^{k}(1)=-3 D^{2} a_{0}^{k}(1) .
\end{aligned}
$$

Besides, due to the symmetry of $a_{0}^{k}(z)$ (symmetric about $z^{0}$ ), if $q \in \mathbb{Z}_{+}$, we have

$$
D^{q} a_{0}^{k}\left(e^{j t_{k+1}}\right)=0 \Longleftrightarrow D^{q} a_{0}^{k}\left(e^{-j t_{k+1}}\right)=0 .
$$

Thus, by a process of row operation (Gaussian elimination), it can be seen that the rank of $\mathbf{A}^{k}$ and $\left(\mathbf{A}^{k} \mid \mathbf{g}\right)$ is $\tau_{0}+\sum_{j=1}^{l} \tau_{j}=n+1$. Since the unknown $\mathbf{h}^{k}$ is a $(n+2) \times 1$ vector, the linear system (35) can be solved containing a free parameter.

Since $\mathbf{h}^{k}$ has been found, let us now fix the desired parameters $\alpha_{0}^{k}, \ldots, \alpha_{n}^{k}$ from $\mathbf{h}^{k}$. In fact, through the substitution in (34), we obtain a linear system $\mathbf{A}_{0} \mathbf{A}_{0}^{k}=\bar{h}^{k}$, where $\mathbf{A}_{0}$ is the coefficient matrix, which is a $(n+2) \times(n+1)$ matrix, $\quad \mathbf{A}_{0}^{k}=\left(\alpha_{0}^{k}, \ldots, \alpha_{n}^{k}\right)^{\top}, \quad \bar{h}^{k}=\left(h_{0}^{k}-\left(\left(1+2 v^{k+1}\right) /(2\right.\right.$ $\left.\left.\left.\left(1+v^{k+1}\right)\right)\right), h_{1}^{k}-9, h_{2}^{k}, \ldots, h_{n+1}^{k}\right)^{\top}$. Note that $h_{0}^{k}, h_{1}^{k}, \ldots, h_{n+1}^{k}$ satisfy $h_{0}^{k}+\sum_{j=1}^{n+1} 2 h_{j}^{k+1}=1$. Then, by the row operation (Gaussian elimination), this linear system is uniquely solvable. In this way, the desired parameters $\alpha_{0}^{k}, \ldots, \alpha_{n}^{k}$ are found containing a free parameter. Similarly, through the second linear system in (33), the parameters $\beta_{0}^{k}, \ldots, \beta_{n-1}^{k}$ can also be uniquely determined. Then, with such chosen parameters, the scheme (10), if convergent, reproduces $\mathrm{EP}_{\Gamma \Lambda_{n}}$.

Remark 1. We denote by $\omega^{k}$ the free parameter obtained in the proof of Theorem 4. For this free parameter, we set $\omega^{k}=(-1)^{n+1} \alpha_{n}^{k}$. Then, if $\omega^{k}$ is chosen to be 0 , the first linear system in (33) can be uniquely solved and the solution can be found with $\alpha_{0}^{k}=1 /\left(4\left(1+v^{k+1}\right)\right), \quad \alpha_{1}^{k}=\cdots=\alpha_{n}^{k}=0$. This solution forces the subsymbol $a_{0}^{k}(z)$ to be $a_{0}^{k}(z)=1$, which implies that the obtained scheme in Theorem 4 is an interpolatory scheme. Besides, with Theorem 2, it can be shown that, with another suitable choice of $\omega^{k}$, this obtained scheme in Theorem 4 also generates $\mathrm{EP}_{\Gamma \Lambda_{n+1}}$ with $\mathrm{EP}_{\Gamma \Lambda_{n}}$ $\subset \mathrm{EP}_{\Gamma \Lambda_{n+1}}$.

Remark 2. For the case $n=0$, the desired scheme reproducing $E P_{\Gamma \Lambda_{0}}$ is actually the scheme (4). From Section 3.1.1, by setting $\omega^{k^{0}}=-\left(\alpha_{0}^{k}-\left(1 / 4\left(1+v^{k+1}\right)\right)\right)$, it reduces to the $\mathrm{D}$ $\mathrm{D}$ 2-point scheme reproducing $\mathrm{EP}_{\Gamma \Lambda_{0}}$ when $\omega^{k}=0$ and becomes the cubic exponential B-spline scheme (1) generating $\mathrm{EP}_{\Gamma \Lambda_{1}}$ and reproducing $\mathrm{EP}_{\Gamma \Lambda_{0}}$ when $\omega^{k}=1 /\left(4\left(1+v^{k+1}\right)\right)$. If, in addition, $\theta^{k}=4 \omega^{k}\left(1+v^{k+1}\right)$, the corresponding $k$-level symbol can be rewritten as $a_{\Gamma \Lambda_{0}, \omega^{k}}^{k}(z)=\left(1-\theta^{k}\right) e_{\Gamma \Lambda_{0}, 1}(z)+\theta^{k} e_{\Gamma \Lambda_{0}, 2}^{k}(z)$, where $e_{\Gamma \Lambda_{0}, 1}(z)$ denotes the symbol of the D-D 2-point scheme and $e_{\Gamma \Lambda_{0}, 2}^{k}(z)$ denotes the $k$-level symbol of the cubic exponential B-spline scheme (1).

In this way, given the set $\Gamma \Lambda_{n}$ with $n \geq 0$, we can derive the desired nonstationary scheme reproducing $\mathrm{EP}_{\Gamma \Lambda_{n}}$, which, in fact, is a quasi-interpolatory scheme combining the interpolatory and noninterpolatory schemes with the same reproduction property. We denote by $\left\{S_{\mathbf{a}^{k}}\right\}_{k \geq 0}$ this newly obtained scheme and by $a_{\Gamma \Lambda_{n}, \omega^{k}}^{k}(z)$ the corresponding $k$-level symbol. In particular, when $n=0,\left\{S_{\mathbf{a}^{k}}\right\}_{k \geq 0}$ refers to the equivalent form of the scheme (4) obtained using the substitution in Remark 2. From the proof of Theorem 4, given a nonstationary scheme with the $k$-level symbol $h^{k}(z)$, which is supported on $[-2 n-2,2 n+2]$ and reproduces $\mathrm{EP}_{\Gamma \Lambda_{n}}$, we can find the parameters $\alpha_{0}^{k}, \ldots, \alpha_{n}^{k}, \beta_{0}^{k}, \ldots, \beta_{n-1}^{k}$ such that $a^{k}(z)$ in (27) satifies (29) $\left(a^{k}(z)\right.$ actually becomes $h^{k}(z)$ ), and thus the corresponding nonstationary scheme reproduces $\mathrm{EP}_{\Gamma \Lambda_{n}}$. This means that the nonstationary scheme with the $k$-level symbol $h^{k}(z)$ falls into the family of the nonstationary schemes in this paper, and these new schemes 
in this paper can actually unify the schemes which are supported on $[-2 n-2,2 n+2]$ and reproduce $\mathrm{EP}_{\Gamma \Lambda_{n}}$.

We point out that the aforementioned process to construct these nonstationary quasi-interpolatory schemes can be generalized to the case of any arity. Note that by the generating function approach, Muntingh [34] derived the expressions for the symbols of the symmetric pseudosplines of any arity. Here, in a similar way, we can also obtain the generalization of these new nonstationary schemes to the case of any arity. Besides, by the same method, we can also get the nonstationary generalization of the work in [34], i.e., the exponential pseudosplines of any arity.

\section{Analysis of Smoothness and Approximation Order}

This section is devoted to the analysis of the convergence, smoothness, and approximation power of the new scheme $\left\{S_{\mathbf{a}_{\Gamma \Lambda_{n}, \omega^{k}}^{k}}\right\}_{k \geq 0}$. Before that, we need to derive the corresponding asymptotical similar scheme first.

In order to derive the asymptotical similar scheme of the scheme $\left\{S_{\mathrm{a}_{\Gamma \Lambda_{n}, \omega^{k}}^{k}}\right\}_{k \geq 0}$, we try to get their symbols. From Remark 2 and Section 3, it can be seen that the symbols of the nonstationary quasi-interpolatory subdivision reproducing $\mathrm{EP}_{\Gamma \Lambda_{0}}$ and $\mathrm{EP}_{\Gamma \Lambda_{1}}$ are actually convex combinations of the symbols of exponential pseudospline schemes. Now, we show that this is true for general $n$. In fact, recall that from Remark 1, the scheme $\left\{S_{\mathbf{a}_{\Gamma \Lambda_{n}, \omega^{k}}^{k}}\right\}_{k \geq 0}$ reduces to the interpolatory scheme reproducing $\mathrm{EP}_{\Gamma \Lambda_{n}}$ when $\omega^{k}=0$ and becomes a noninterpolatory one generating $\mathrm{EP}_{\Gamma \Lambda_{n+1}}$ and reproducing $\mathrm{EP}_{\Gamma \Lambda_{n}}$ with $\Gamma \Lambda_{n} \subset \Gamma \Lambda_{n+1}$ when $\omega^{k}$ is chosen to be another suitable value. For these two schemes, let $e_{\Gamma \Lambda_{n}, 1}^{k}=$ $\left\{e_{1,-2 n-1}^{k}, 0, \ldots, 0, e_{1,-1}^{k}, 1, e_{1,1}^{k}, 0 \ldots, 0, e_{1,2 n+1}^{k}\right\} \quad$ and $\quad e_{\Gamma \Lambda_{n}, 2}^{k}=$ $\left\{e_{1,-2 n-2}^{k}, e_{1,-2 n-1}^{k}, \ldots, e_{1,2 n+1}^{k}, e_{1,2 n+2}^{k}\right\}$ denote their $k$-level masks, and by $e_{\Gamma \Lambda_{n}, 1}^{k}(z)$ and $e_{\Gamma \Lambda_{n}, 2}^{k}(z)$ we denote their corresponding $k$-level symbols. In particular, when $n=1$, these two $k$-level symbols are just the pair of symbols in (23). Then, from ([33], Section 5), these two schemes are just the odd symmetric exponential pseudospline schemes (see [33] for the way to compute their symbols). In this way, the corresponding $k$-level symbol $a_{\Gamma \Lambda_{n}, \omega^{k}}^{k}(z)$ is actually a convex combination of $e_{\Gamma \Lambda_{n}, 1}^{k}(z)$ and $e_{\Gamma \Lambda_{n}, 2}^{k}(z)$. In other words, we have the following result.

Lemma 2. Given the set $\Gamma \Lambda_{n}$, the k-level symbol $a_{\Gamma \Lambda_{n}, \omega^{k}}^{k}(z)$ of the new scheme $\left\{S_{\mathbf{a}_{\Gamma \Lambda_{n}, \omega^{k}}^{k}}\right\}_{k \geq 0}$ can be written as

$$
a_{\Gamma \Lambda_{n}, \omega^{k}}^{k}(z)=\left(1-\theta^{k}\right) e_{\Gamma \Lambda_{n}, 1}^{k}(z)+\theta^{k} e_{\Gamma \Lambda_{n}, 2}^{k}(z),
$$

where $\theta^{k}$ is a free parameter depending on $k$.

Remark 3. Since the exponential pseudospline schemes in [33] are derived using polynomial correction, Theorem 4 and Lemma 2 imply that quite a number of the nonstationary subdivision schemes obtained using the polynomial correction, including the existing interpolatory schemes reproducing exponential polynomials, such as the ones in $[12,25]$, can also be derived by suitably using the push-back operation.

Lemma 2 also implies that these nonstationary quasiinterpolatory subdivision schemes are also parameter-dependent schemes (see also [27]). Now, we let $\theta^{k}=\omega^{k} /\left|e_{2,2 n+2}^{k}\right|=\omega^{k} /\left|e_{2,-2 n-2}^{k}\right|$ in Lemma 2. As a result, we choose $\omega^{k}=\left|e_{2,2 n+2}^{k}\right|$ in the new scheme $\left\{S_{\mathrm{a}_{\Gamma \Lambda_{n}, \omega^{k}}^{k}}\right\}_{k \geq 0}$ so that it also generates $\mathrm{EP}_{\Gamma \Lambda_{n+1}}$. Now, we make a mild condition on the free parameter $\omega^{k}$. We assume that the limit of $\omega^{k}$ as $k$ tends to infinity exists and we denote its limit by $\omega$, that is to say we assume $\lim _{k \rightarrow \infty} \omega^{k}:=\omega \in \mathbb{R}$ exists. Then, together with Lemma 2, we can show that the asymptotical similar scheme of $\left\{S_{\mathbf{a}_{\Gamma A_{n, a}, k^{k}}^{k}}\right\}_{k \geq 0}$ is the scheme $S_{2 M}$ with $M=n+1$ in [26]. This is shown in the following result.

Proposition 1. Assume $\lim _{k \longrightarrow \infty} \omega^{k}:=\omega \in \mathbb{R}$. ien, the asymptotical similar scheme of the new nonstationary scheme $\left\{S_{\mathrm{a}_{\Gamma \Lambda_{n}, \omega^{k}}^{k}}\right\}_{k \geq 0}$ reproducing $\mathrm{EP}_{\Gamma \Lambda_{n}}$ is the stationary scheme $S_{2 M}$ with $M=n+1$.

Proof. Due to the fact that the asymptotical similar counterparts of the exponential pseudosplines are the pseudosplines [33], we have

$$
\begin{aligned}
& \lim _{k \longrightarrow \infty} e_{\Gamma \Lambda_{n}, 1}^{k}(z)=a_{n+1}^{n}(z), \\
& \lim _{k \longrightarrow \infty} e_{\Gamma \Lambda_{n}, 2}^{k}(z)=a_{n+2}^{n}(z),
\end{aligned}
$$

where

$$
\begin{aligned}
a_{m}^{l}(z)= & 2\left(\frac{(1+z)^{2}}{4 z}\right)^{m} \sum_{i=0}^{l}\left(\begin{array}{c}
m+l \\
i
\end{array}\right) \\
& \cdot\left(-\frac{(1-z)^{2}}{4 z}\right)^{i}\left(\frac{(1+z)^{2}}{4 z}\right)^{l-i}, \quad m, l \in \mathbb{N}_{0},
\end{aligned}
$$

denotes the pseudosplines of type II [35]. In this way, for the leading term of $a_{n+2}^{n}(z)$, the absolute value of its coefficient is $\lim _{k \longrightarrow \infty}\left|e_{2,2 n+2}^{k}\right|=\left(\begin{array}{c}2 n+2 \\ n+1\end{array}\right) / 16^{n+1}$. Since $\theta^{k}=\omega^{k} /\left|e_{2,2 n+2}^{k}\right|$, it can be seen that $\lim _{k \longrightarrow \infty} \theta^{k}=\omega\left(16^{n+1} /\left(\begin{array}{c}2 n+2 \\ n+1\end{array}\right)\right)$ and we denote this limit by $\theta$. Thus, we have

$$
a_{n, \omega}(z):=\lim _{k \longrightarrow \infty} a_{\Gamma \Lambda_{n}, \omega^{k}}^{k}(z)=(1-\theta) a_{n+1}^{n}(z)+\theta a_{n+2}^{n}(z) .
$$

Therefore, $a_{n, \omega}(z)$ is just the symbol of the scheme $S_{2 M}$ with $M=n+1$ in [26] (see [35]). Thus, the scheme $S_{2 M}$ with 
$M=n+1$ in [26] is the asymptotical similar scheme of $\left\{S_{\mathbf{a}_{\Gamma \Lambda_{n}, \omega^{k}}^{k}}\right\}_{k \geq 0}$.

Based on Proposition 1, now we investigate the $C^{r}$ convergence of the scheme $\left\{S_{\mathbf{a}_{\Gamma \Lambda_{n}, \omega^{k}}^{k}}\right\}_{k \geq 0}$. In fact, we have the following result.

Theorem 5. Given the set $\Gamma \Lambda_{n}$, for the new scheme $\left\{S_{\mathbf{a}^{k} \Lambda_{n, \omega^{k}}}\right\}_{k \geq 0}$, it is $C^{r}$ convergent with $r<2 n+4$, if the free parameter $\omega^{k}$ is such that the asymptotical similar counterpart is $C^{r}$ convergent.

Proof. From Theorem 4 and Remark 1, the new scheme $\left\{S_{\mathbf{a}^{k} \Lambda_{n}, \omega^{k}}\right\}_{k \geq 0}$ reproduces $\operatorname{EP}_{\Gamma \Lambda_{n}}$ and generates $\mathrm{EP}_{\Gamma \Lambda_{n+1}}$ with $\Gamma \Lambda_{n} \subset \Gamma \Lambda_{n+1}$. Then, according to [36], Corollary 14, this new nonstationary scheme satisfies approximate sum rules of order $\tilde{r}$ with $\tilde{r}=2 \tau_{0}+2 \sum_{j=1}^{l} \tau_{l}=2[(n+1)+1]=2 n+4$. Then, from Theorem 1 , this new nonstationary scheme is $C^{r}$ convergent, if the free parameter $\omega^{k}$ is such that the asymptotical similar subdivision is $C^{r}$ convergent with $r<2 n+4$.

Now, let us discuss the approximation order of the scheme $\left\{S_{\mathbf{a}^{k} \Lambda_{n, \omega^{k}}}\right\}_{k \geq 0}$. Let $f$ be a function satisfying $\left\|D^{j} f\right\|_{L_{\infty}(K)}<\infty$ with $j=0, \ldots, L, L=2 n+2$ and $K$ being a compact set in $\mathbb{R}$. Assume that the initial data is of the form $\mathbf{f}^{\kappa}:=\left\{f_{j}^{\kappa}=f\left(j 2^{-\kappa}\right), j \in \mathbb{Z}\right\}$ for some $\kappa \in \mathbb{Z}_{+}$. Then, for the scheme $\left\{S_{\mathbf{a}^{k} \Lambda_{n}, \omega^{k}}\right\}_{k \geq 0}$, according to [36], Theorem 21 (see also [37]), we have

$$
\left\|f^{\infty}-f\right\|_{L_{\infty}(K)} \leq c_{f} 2^{-\kappa L},
$$

where $f^{\infty}$ denotes the limit of the scheme $\left\{S_{\mathbf{a}_{\Gamma A_{n}, \omega^{k}}^{k}}\right\}_{k \geq 0}$ obtained from $\mathbf{f}^{\kappa}$ and $c_{f}$ is a positive constant only dependent on $f$. This implies that this newly obtained scheme $\left\{S_{\mathbf{a}^{k} \Lambda_{n, \omega^{k}}}\right\}_{k \geq 0}$ has the same approximation order as its stationary counterpart (see [26] for the approximation order of the corresponding stationary counterpart).

\section{Examples and Comparison}

This section is devoted to the comparison with the exponential pseudospline schemes and several examples and to illustrate the performance of this new family of nonstationary schemes.

Note that the exponential pseudospline schemes contain the interpolatory exponentials reproducing schemes and the exponential B-spline schemes as special subclasses. Thus, now we first compare the new nonstationary quasi-interpolatory schemes with the interpolatory ones reproducing the same exponential polynomials. Given the set $\Gamma \Lambda_{n}$, recall that the new scheme $\left\{S_{a_{\Gamma \Lambda_{n}, \omega^{k}}^{k}}\right\}_{k \geq 0}$ reproduces $\operatorname{EP}_{\Gamma \Lambda_{n}}$ and is supported on $[-2 n-2,2 n+2]$. From Remark 1, when $\omega^{k}=0$, this scheme becomes the interpolatory subdivision with the same reproduction property, which is actually supported on $[-2 n-1,2 n+1]$. Besides, Theorem 6 implies that this newly obtained scheme has the same smoothness order as its stationary counterpart. Thus, we can choose $\omega^{k}$ away from 0 to increase its smoothness order at the expense of slightly larger support. This is shown by Table 1, which gives the smoothness orders of this new nonstationary scheme between the cases $\omega^{k}=0$ and $\omega^{k} \neq 0$ (maximal smoothness order).

That the scheme $\left\{S_{\mathbf{a}_{\Gamma \Lambda_{n}, \omega^{k}}^{k}}\right\}_{k \geq 0}$ can reach a higher smoothness order by choosing $\omega^{k}$ away from 0 is also reflected by Table 2. Table 2 gives the comparison among the cubic exponential B-spline scheme (1), the scheme $\left\{S_{\mathbf{a}_{\Gamma \Lambda_{1}, 0}^{k}}\right\}_{k \geq 0}$ (i.e., the nonstationary interpolatory 4-point scheme in [25]), and the scheme $\left\{S_{\mathbf{a}_{\Gamma_{1}, \omega}^{k}}\right\}_{k \geq 0}$ on the support, the space they reproduce, the approximation order, and the smoothness order. From Table 2, it can be seen that with only a slight increase of the support, the scheme $\left\{S_{\mathbf{a}_{\Gamma \Lambda_{1}, \omega^{k}}}\right\}_{k \geq 0}$ can reach the highest smoothness order among these three schemes and higher approximation order than the cubic exponential B-spline scheme (1). Besides, it also implies that the new schemes in this paper have much higher approximation order than the exponential B-spline schemes due to the reproduction property of these two families of schemes.

From Lemma 2 and Theorem 4, the new scheme $\left\{S_{\mathbf{a}^{k} \Lambda_{n}, \omega^{k}}\right\}_{k \geq 0}$ also contains a noninterpolatory scheme besides the interpolatory one with the same reproduction property. Compared with this noninterpolatory scheme, the scheme $\left\{S_{\mathbf{a}^{k} \Lambda_{n}, \omega^{k}}\right\}_{k \geq 0}$ has at least the same smoothness order. Besides, due to the existence of the parameter $\omega^{k}$, the scheme $\left\{S_{\mathbf{a}_{\Gamma \Lambda_{n}, \omega^{k}}^{k}}\right\}_{k \geq 0}$ differs with different values of $\omega^{k}$ but with the same reproduction property. Also, the parameter $\omega^{k}$ makes the scheme $\left\{S_{\mathbf{a}^{k} \Lambda_{n}, \omega^{k}}\right\}_{k \geq 0}$ have better flexibility than the exponential pseudosplines with the same reproduction property in curve design, which is reflected by Figures 1 and 2 obtained by the scheme in the following example 2 .

Now, we present some concrete examples to illustrate the performance of this new family of nonstationary schemes.

Example 1. When $n=0$, from Remark 2, the new nonstationary scheme $\left\{S_{\mathbf{a}_{\Gamma \Lambda_{0}, \omega^{k}}^{k}}\right\}_{k \geq 0}$ reproducing $\mathrm{EP}_{\Gamma \Lambda_{0}}$ is actually the scheme (4). From Theorem 1, it is $C^{r}$ convergent if $\omega^{k}$ is such that the corresponding stationary scheme is $C^{r}$ convergent with $r=0,1,2$.

Example 2. When $n=1$, the new scheme $\left\{S_{\mathbf{a}^{k} \Lambda_{1}, \omega^{k}}\right\}_{k \geq 0}$ is just the scheme with the $k$-level symbol in (21). Theorem 1 implies that this new scheme is $C^{r}$ convergent if $\omega^{k}$ is such that the asymptotical similar scheme is $C^{r}$ convergent with $r=0,1,2,3$. 
TABLE 1: Comparison on the smoothness orders of the scheme $\left\{S_{\mathfrak{a}_{\Gamma \Lambda_{n}, \omega^{k}}^{k}}\right\}_{k \geq 0}$ reproducing $\mathrm{EP}_{\Gamma \Lambda_{n}}$ with different $n$ between the cases $\omega^{k}=0$ and $\omega^{k} \neq 0$ (maximal smoothness order).

\begin{tabular}{lccccccc}
\hline & $n=0$ & $n=1$ & $n=2$ & $n=3$ & $n=4$ & $n=5$ & $n=6$ \\
\hline$\omega^{k}=0$ & 0 & 1 & 2 & 3 & 4 & 4 \\
$\omega^{k} \neq 0$ & 2 & 3 & 4 & 5 & 6 & 6 & 7 \\
\hline
\end{tabular}

TABLE 2: Comparison between the scheme $\left\{S_{\mathbf{a}_{\Gamma_{1}, \omega^{k}}^{k}}\right\}_{k \geq 0}$ in cases $\omega^{k}=0$ and $\omega^{k} \neq 0$ and the cubic exponential B-spline scheme (1) on the support, the space they reproduce, the approximation order, and the smoothness order.

\begin{tabular}{|c|c|c|c|c|}
\hline Subdivision & Support & Space reproduced & Approximation order & Smoothness order \\
\hline$\left\{S_{\mathbf{a}_{\Gamma \Lambda_{1}, \omega^{k}}^{k}}\right\}_{k \geq 0}$ & {$[-4,4]$} & $\mathrm{EP}_{\Gamma \Lambda_{1}}$ & 4 & 3 \\
\hline Scheme (1) & {$[-2,2]$} & $\mathrm{EP}_{\Gamma \Lambda_{0}}$ & 2 & 2 \\
\hline$\left\{S_{\mathbf{a}_{1,0}^{k}}\right\}_{k \geq 0}$ & {$[-3,3]$} & $\mathrm{EP}_{\Gamma \Lambda_{1}}$ & 4 & 1 \\
\hline
\end{tabular}

Example 3. When $n=2$, let $\Gamma \Lambda_{2}=\{(0,2),( \pm t, 1)$, $( \pm 2 t, 1)\}$. Then, we have

$$
\mathrm{EP}_{\Gamma \Lambda_{2}}=\left\{1, x, e^{ \pm \mathrm{tx}}, e^{ \pm 2 \mathrm{tx}}\right\}
$$

From Theorem 4 , by suitably choosing $\alpha_{0}^{k}, \alpha_{1}^{k}, \alpha_{2}^{k}$ and $\beta_{0}^{k}, \beta_{1}^{k}$, we can derive the new nonstationary quasi-interpolatory scheme $\left\{S_{a_{\Gamma \Lambda_{2}, \omega^{k}}^{k}}\right\}_{k \geq 0}$ reproducing the space $\mathrm{EP}_{\Gamma \Lambda_{2}}$ in (44), and the corresponding $k$-level symbol can be written as

$$
a_{\Gamma \Lambda_{2}, \omega^{k}}^{k}(z)=\left(1-\theta^{k}\right) e_{\Gamma \Lambda_{2}, 1}^{k}(z)+\theta^{k} e_{\Gamma \Lambda_{2}, 2}^{k}(z) .
$$

Here, $e_{\Gamma \Lambda_{2}, 1}^{k}(z)$ is the $k$-level symbol of the interpolatory scheme reproducing the space in (44) ([12], Section 4.2):

$$
\begin{aligned}
e_{\Gamma \Lambda_{2}, 2}^{k}(z)= & \frac{(1+z)^{4}}{8} \frac{z^{2}+2 v^{k+1} z+1}{2\left(v^{k+1}+1\right)} \frac{z^{2}+\left(4\left(v^{k+1}\right)^{2}-2\right) z+1}{4\left(v^{k+1}\right)^{2}} \\
& \cdot\left(v_{1}^{k}\left(1+z^{4}\right)+v_{2}^{k}\left(z+z^{3}\right)+\left(1-2 v_{1}^{k}-2 v_{2}^{k}\right) z^{2}\right) z^{-6},
\end{aligned}
$$

with

$$
\left\{\begin{array}{l}
v_{1}^{k}=\frac{4\left(v^{k+1}\right)^{4}+10\left(v^{k+1}\right)^{3}+4\left(v^{k+1}\right)^{2}+v^{k+1}+1}{8\left(v^{k+1}\right)^{2}\left(v^{k+1}+1\right)^{2}\left(2 v^{k+1}-1\right)\left(2\left(v^{k+1}\right)^{2}-1\right)} \\
v_{2}^{k}=-\frac{8\left(v^{k+1}\right)^{6}+24\left(v^{k+1}\right)^{5}+14\left(v^{k+1}\right)^{4}+4\left(v^{k+1}\right)^{3}+3\left(v^{k+1}\right)^{2}+2 v^{k+1}+1}{4\left(v^{k+1}\right)^{2}\left(2 v^{k+1}-1\right)\left(2\left(v^{k+1}\right)^{2}-1\right)\left(1+v^{k+1}\right)^{2}}
\end{array}\right.
$$

being the $k$-level symbol of the scheme generating $\mathrm{EP}_{\Gamma \Lambda_{3}}:=\left\{1, x, x^{2}, x^{3}, e^{ \pm \mathrm{tx}}, e^{ \pm 2 \mathrm{tx}}\right\}$ with $\Gamma \Lambda_{3}=\{(0,4),( \pm t, 1)$, $( \pm 2 t, 1)\}$ and reproducing the space in (44) and $\theta^{k}=\left(\left(512\left(v^{k+1}+1\right)^{3}\left(v^{k+1}\right)^{4} \quad\left(2 v^{k+1}-1\right)\left(2\left(v^{k+1}\right)^{2}-1\right)\right) /\right.$ $\left.\left(4\left(v^{k+1}\right)^{4}+10\left(v^{k+1}\right)^{3}+4\left(v^{k+1}\right)^{2}+v^{k+1}+1\right)\right) \omega^{k}$.

From Theorem 5, this new scheme is $C^{r}$ convergent if the parameter $\omega^{k}$ is such that the asymptotical similar counterpart is $C^{r}$ convergent with $r=0,1,2,3,4$.

Example 4. When $n=3$, let $\Gamma \Lambda_{3}:=\{(0,2),( \pm t, 1)$, $( \pm 2 t, 1),( \pm 3 t, 1)\}$, and then we have

$$
\mathrm{EP}_{\Gamma \Lambda_{3}}=\left\{1, x, e^{ \pm \mathrm{tx}}, e^{ \pm 2 \mathrm{tx}}, e^{ \pm 3 \mathrm{tx}}\right\} .
$$

From Theorem 4, with a suitable choice of the parameters $\alpha_{0}^{k}, \ldots, \alpha_{3}^{k}$ and $\beta_{0}^{k}, \beta_{1}^{k}, \beta_{2}^{k}$, we can derive the scheme $\left\{S_{\mathbf{a}^{k} \Lambda_{3}, \omega^{k}}\right\}_{k \geq 0}$ reproducing the space in (48) with the $k$-level symbol

$$
a_{\Gamma \Lambda_{3}, \omega^{k}}^{k}(z)=\left(1-\theta^{k}\right) e_{\Gamma \Lambda_{3}, 1}^{k}(z)+\theta^{k} e_{\Gamma \Lambda_{3}, 2}^{k}(z) .
$$

Here, $e_{\Gamma \Lambda_{3}, 1}^{k}(z)$ is the $k$-level symbol of the scheme (32)(33) in [24]: 


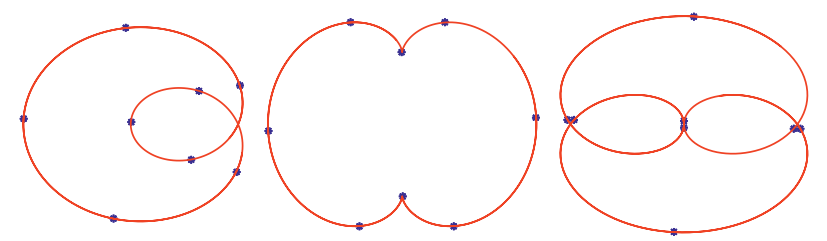

FIGURE 3: Epitrochoid curves reproduced by the scheme $\left\{S_{\mathfrak{a}_{\Gamma \Lambda_{3}, \omega^{k}}^{k}}\right\}_{k \geq 0}$ reproducing the space in (48) with $\omega^{k}=0.002$ and $v^{0}=\cos (\pi / 4)$.

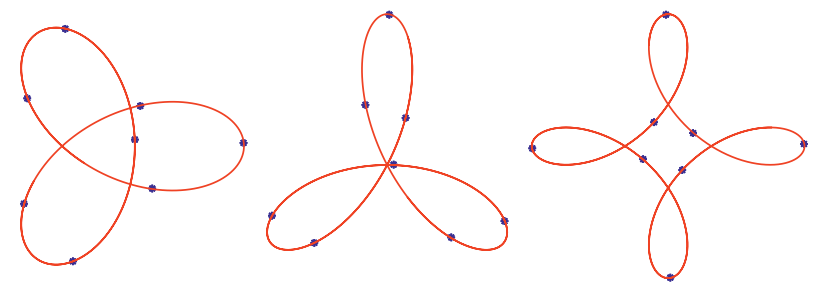

FIGURE 4: Hypotrochoid curves reproduced by the scheme $\left\{S_{\mathfrak{a}_{\mathrm{\Gamma} \Lambda_{3}, \omega^{k}}^{k}}\right\}_{k \geq 0}$ reproducing the space in (48) with $\omega^{k}=0.002$ and $v^{0}=\cos (\pi / 4)$.

TABLE 3: The schemes in the above 4 examples and the different properties.

\begin{tabular}{|c|c|c|c|c|c|}
\hline Subdivision & Support & Smooth. order & Dim. space. generated & Dim. space. reproduced & Approxi. order \\
\hline$\left\{S_{\mathbf{a}^{k}}\right\}_{k \geq 0}$ & {$[-2,2]$} & 2 & 4 & 2 & 2 \\
\hline$\left\{S_{\mathbf{a}^{k}}\right.$ & {$[-4,4]$} & 3 & 6 & 4 & 4 \\
\hline$\left\{S_{\mathbf{a}^{k}}\right\}_{k \geq 0}$ & {$[-6,6]$} & 4 & 8 & 6 & 6 \\
\hline$\left\{S_{\mathbf{a}_{\Gamma \Lambda_{3}, \omega^{k}}}\right\}_{k \geq 0}$ & {$[-8,8]$} & 5 & 10 & 8 & 8 \\
\hline
\end{tabular}

$$
\begin{aligned}
e_{\Gamma \Lambda_{3}, 2}^{k}(z)= & \frac{(1+z)^{4}}{8} \frac{z^{2}+2 v^{k+1} z+1}{2+2 v^{k+1}} \\
& \cdot \frac{z^{2}+\left(4\left(v^{k+1}\right)^{2}-2\right) z+1}{4\left(v^{k+1}\right)^{2}} \\
& \cdot \frac{z^{2}+2 v^{k+1}\left(4\left(v^{k+1}\right)^{2}-3\right) z+1}{2+2 v^{k+1}\left(4\left(v^{k+1}\right)^{2}-3\right)} q^{k}(z) z^{-8},
\end{aligned}
$$

where $q^{k}(z)=r^{k}\left(1+z^{6}\right)+s^{k}\left(z+z^{5}\right)+t^{k}\left(z^{2}+z^{4}\right)+(1-$ $\left.2 r^{k}-2 s^{k}-2 t^{k}\right) z^{3}$ with $r^{k}, s^{k}$, and $t^{k}$ satisfying

$$
\begin{aligned}
e_{\Gamma \Lambda_{3}, 2}^{k}(1) & =e_{\Gamma \Lambda_{3}, 2}^{k}\left(e^{ \pm t_{k+1}}\right)=e_{\Gamma \Lambda_{3}, 2}^{k}\left(e^{ \pm 2 t_{k+1}}\right) \\
& =e_{\Gamma \Lambda_{3}, 2}^{k}\left(e^{ \pm 3 t_{k+1}}\right)=2, \\
D^{1} e_{\Gamma \Lambda_{3}, 2}^{k}(1) & =0
\end{aligned}
$$

and $\theta^{k}=\omega^{k} /\left|\gamma^{k}\right|$ with $\gamma^{k}$ being the coefficient of the leading term of $e_{\Gamma \Lambda_{3}, 2}^{k}(z)$. Note that the scheme with the $k$-level symbol $e_{\Gamma \Lambda_{3}, 2}^{k}(z)$ also generates $\mathrm{EP}_{\Gamma \Lambda_{4}}=\left\{1, x, x^{2}, x^{3}, e^{ \pm \mathrm{tx}}\right.$, $\left.e^{ \pm 2 \mathrm{tx}}, e^{ \pm 3 \mathrm{tx}}\right\}$ with $\Gamma \Lambda_{4}=\{(0,4),( \pm t, 1),( \pm 2 t, 1),( \pm 3 t, 1)\}$.

From Theorem 5, it can be seen that this nonstationary scheme is $C^{r}$ convergent if $\omega^{k}$ is such that the asymptotical similar counterpart is $C^{r}$ convergent with $r=0,1, \ldots, 5$.

Figures 3 and 4 show some epitrochoid curves and hypotrochoid curves reproduced by the scheme $\left\{S_{\mathrm{a}_{\Gamma \Lambda_{3}, \omega^{k}}}\right\}_{k \geq 0}$ reproducing the space in (48) with $v^{0}=\cos (\pi / 4)$ and $\omega^{k}=0.002$.

Table 3 lists the schemes $\left\{S_{\mathbf{a}^{k} \Lambda_{n, \omega} k}\right\}_{k \geq 0}$ with $n=0,1,2,3$ in the aforementioned examples on the support, smoothness order (smooth. order), dimension of the exponential polynomial space generated (dim. space. generated), dimension of the exponential polynomial space reproduced (dim. space. reproduced), and the approximation order (approxi. order). From Table 3, we can see the change of these properties of the scheme $\left\{S_{\mathbf{a}_{\Gamma \Lambda_{n}, \omega^{k}}^{k}}\right\}_{k \geq 0}$ with the change of $n$. 


\section{Conclusion}

This paper presents a new family of nonstationary subdivision schemes by suitably using the so-called push-back operation. Each one of these new schemes is a nonstationary quasi-interpolatory subdivision and reproduces a certain exponential polynomial space. These new schemes unify and extend the existing interpolatory schemes with the same reproduction property and the noninterpolatory ones like the cubic exponential B-spline scheme. We show that they have higher smoothness orders than the interpolatory ones with the same reproduction property and better accuracy than the exponential B-spline schemes. Note that this new family of nonstationary schemes are actually constant reproducing nonstationary schemes and their symbols are odd symmetric. Therefore, future works may focus on the investigation of the generalization of the schemes in this paper, which can reproduce even more exponential polynomials. Besides, the basic limit functions of these new schemes satisfy nonstationary refinable equations, which are the key ingredients to derive the nonstationary multiresolution analysis (see also [38]). Thus, future works can also focus on the construction of nonstationary (biorthogonal) wavelets using these new schemes. Similar works include the generalized Daubechies wavelets in [4] and the biorthogonal wavelets in [3].

\section{Data Availability}

The data used to support the findings of this study are available from the corresponding author upon request.

\section{Conflicts of Interest}

The authors declare that they have no conflicts of interest.

\section{Acknowledgments}

This study was supported by the National Science Foundation for Young Scientists of China (grant no. 61803129).

\section{References}

[1] J. Shen, J. Kosinka, M. Sabin, and N. Dodgson, "Converting a CAD model into a non-uniform subdivision surface," Computer Aided Geometric Design, vol. 48, pp. 17-35, 2016.

[2] G. Lavoué, F. Dupont, and A. Baskurt, "A framework for quad/triangle subdivision surface fitting: application to mechanical objects," Computer Graphics Forum, vol. 26, no. 1, pp. 1-14, 2007.

[3] H. O. Kim, R. Y. Kim, Y. J. Lee, and J. Yoon, "Quasi-interpolatory refinable functions and construction of biorthogonal wavelet systems," Advances in Computational Mathematics, vol. 33, no. 3, pp. 255-283, 2010.

[4] N. Dyn, O. Kounchev, D. Levin, and H. Render, "Regularity of generalized Daubechies wavelets reproducing exponential polynomials with real-valued parameters," Applied and Computational Harmonic Analysis, vol. 37, no. 2, pp. 288-306, 2014.

[5] A. Badoual, P. Novara, L. Romani, D. Schmitter, and M. Unser, "A non-stationary subdivision scheme for the construction of deformable models with sphere-like topology," Graphical Models, vol. 94, pp. 38-51, 2017.

[6] D. Burthar, B. Hamann, and G. Umlauf, "Iso-geometric finite element analysis based on Catmull-Clark: subdivision solids," Computer Graphics Forum, vol. 29, no. 5, pp. 1575-1584, 2010.

[7] N. Dyn, "Subdivision schemes in CAGD," Advances in Numerical Analysis, vol. 2, pp. 36-104, 1992.

[8] A. S. Cavaretta, W. Dahmen, and C. A. Micchelli, "Stationary subdivision," Memoirs of the American Mathematical Society, vol. 453, pp. 1-185, 1991.

[9] B.-J. Li, Z.-L. Yu, B.-W. Yu, Z.-X. Su, and X.-P. Liu, "Nonstationary subdivision for exponential polynomials reproduction," Acta Mathematicae Applicatae Sinica, English Series, vol. 29, no. 3, pp. 567-578, 2013.

[10] Y. J. Lee and J. Yoon, "Non-stationary subdivision schemes for surface interpolation based on exponential polynomials," Applied Numerical Mathematics, vol. 60, no. 1-2, pp. 130-141, 2010.

[11] M. Asghar and G. Mustafa, "Family of $a$-ary univariate subdivision schemes generated by laurent polynomial," Mathematical Problems in Engineering, vol. 2018, Article ID 7824279, 11 pages, 2018.

[12] L. Romani, "From approximating subdivision schemes for exponential splines to high-performance interpolating algorithms," Journal of Computational and Applied Mathematics, vol. 224, no. 1, pp. 383-396, 2009.

[13] C. Conti, L. Gemignani, and L. Romani, "From approximating to interpolatory non-stationary subdivision schemes with the same generation properties," Advances in Computational Mathematics, vol. 35, no. 2-4, pp. 217-241, 2011.

[14] B. Jeong, Y. J. Lee, and J. Yoon, "A family of non-stationary subdivision schemes reproducing exponential polynomials," Journal of Mathematical Analysis and Applications, vol. 402, no. 1, pp. 207-219, 2013.

[15] C. Conti and L. Romani, "Affine combination of B-spline subdivision masks and its non-stationary counterparts," BIT Numerical Mathematics, vol. 50, no. 2, pp. 269-299, 2010.

[16] C. V. Beccari, G. Casciola, and L. Romani, "A unified framework for interpolating and approximating univariate subdivision," Applied Mathematics and Computation, vol. 216, no. 4, pp. 1169-1180, 2010.

[17] G. Li and W. Ma, "A method for constructing interpolatory subdivision schemes and blending subdivisions," Computer Graphics Forum, vol. 26, no. 2, pp. 185-201, 2007.

[18] J. Maillot and J. Stam, "A unified subdivision scheme for polygonal modeling," Computer Graphics Forum, vol. 20, no. 3, pp. 471-479, 2001.

[19] S. Lin, F. You, X. Luo, and Z. Li, "Deducing interpolating subdivision schemes from approximating subdivision schemes," ACM Transactions on Graphics, vol. 27, no. 5, pp. 1-7, 2008.

[20] Z. Luo and W. Qi, "On interpolatory subdivision from approximating subdivision scheme," Applied Mathematics and Computation, vol. 220, pp. 339-349, 2013.

[21] H. Zhang and G. Wang, "Semi-stationary push-back subdivision schemes," Journal of Software, vol. 13, no. 9, pp. 1830-1839, 2002.

[22] J. Shi, J. Tan, Z. Liu, and L. Zhang, "Six-point subdivision schemes with cubic precision," Mathematical Problems in Engineering, vol. 2018, Article ID 2324893, 9 pages, 2018.

[23] Z. Shi, S. Lin, X. Luo, and R. Wang, "Interpolatory and mixed loop schemes," Computer Graphics Forum, vol. 27, no. 7, pp. 1829-1835, 2008. 
[24] H. Zheng and B. Zhang, "A non-stationary combined subdivision scheme generating exponential polynomials," $A p$ plied Mathematics and Computation, vol. 313, pp. 209-221, 2017.

[25] C. Beccari, G. Casciola, and L. Romani, "A non-stationary uniform tension controlled interpolating 4-point scheme reproducing conics," Computer Aided Geometric Design, vol. 24, no. 1, pp. 1-9, 2007.

[26] S. W. Choi, B.-G. Lee, Y. J. Lee, and J. Yoon, "Stationary subdivision schemes reproducing polynomials," Computer Aided Geometric Design, vol. 23, no. 4, pp. 351-360, 2006.

[27] M. Charina, C. Conti, N. Guglielmi, and V. Protasov, "Limits of level and parameter dependent subdivision schemes: a matrix approach," Applied Mathematics and Computation, vol. 272, pp. 20-27, 2016.

[28] N. Dyn and D. Levin, "Subdivision schemes in geometric modelling," Acta Numerica, vol. 11, pp. 73-144, 2002.

[29] C. Conti, N. Dyn, C. Manni, and M.-L. Mazure, "Convergence of univariate non-stationary subdivision schemes via asymptotic similarity," Computer Aided Geometric Design, vol. 37, pp. 1-8, 2015.

[30] M. Charina, C. Conti, N. Guglielmi, and V. Protasov, "Regularity of non-stationary subdivision: a matrix approach," Numerische Mathematik, vol. 135, no. 3, pp. 639678, 2017.

[31] C. Conti and L. Romani, "Algebraic conditions on non-stationary subdivision symbols for exponential polynomial reproduction," Journal of Computational and Applied Mathematics, vol. 236, no. 4, pp. 543-556, 2011.

[32] M. Charina, C. Conti, and L. Romani, "Reproduction of exponential polynomials by multivariate non-stationary subdivision schemes with a general dilation matrix," Numerische Mathematik, vol. 127, no. 2, pp. 223-254, 2014.

[33] C. Conti, L. Gemignani, and L. Romani, "Exponential pseudosplines: looking beyond exponential B-splines," Journal of Mathematical Analysis and Applications, vol. 439, no. 1, pp. 32-56, 2016.

[34] G. Muntingh, "Symbols and exact regularity of symmetric pseudo-splines of any arity," BIT Numerical Mathematics, vol. 57, no. 3, pp. 867-900, 2017.

[35] N. Dyn, K. Hormann, M. A. Sabin, and Z. Shen, "Polynomial reproduction by symmetric subdivision schemes," Journal of Approximation Theory, vol. 155, no. 1, pp. 28-42, 2008.

[36] C. Conti, L. Romani, and J. Yoon, "Approximation order and approximate sum rules in subdivision," Journal of Approximation Theory, vol. 207, pp. 380-401, 2016.

[37] B. Jeong, H. O. Kim, Y. J. Lee, and J. Yoon, "Exponential polynomial reproducing property of non-stationary symmetric subdivision schemes and normalized exponential Bsplines," Advances in Computational Mathematics, vol. 38, no. 3, pp. 647-666, 2013.

[38] A. Cohen and N. Dyn, "Nonstationary subdivision schemes, multiresolution analysis, and wavelet packets," Wavelet Analysis and Its Applications, vol. 7, pp. 189-200, 1998. 


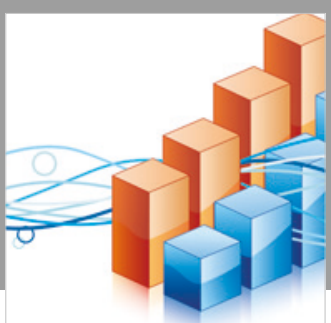

Advances in

Operations Research

\section{-n-m}
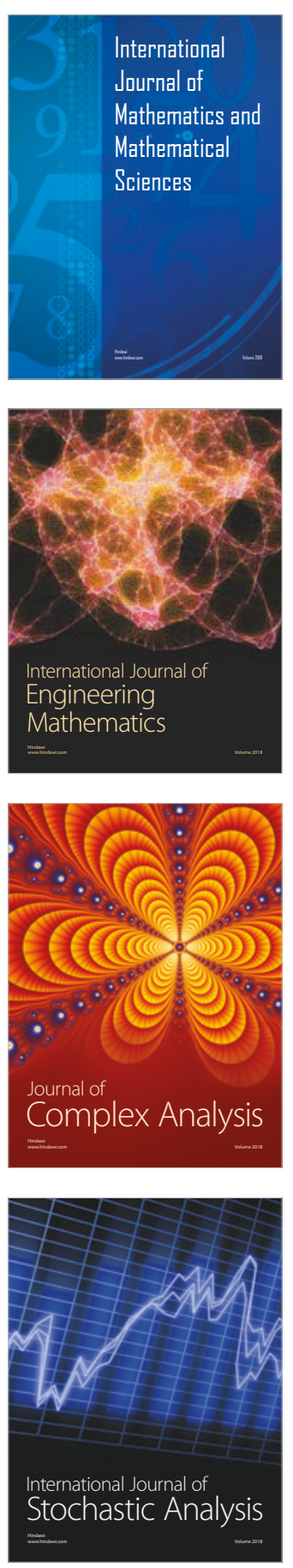
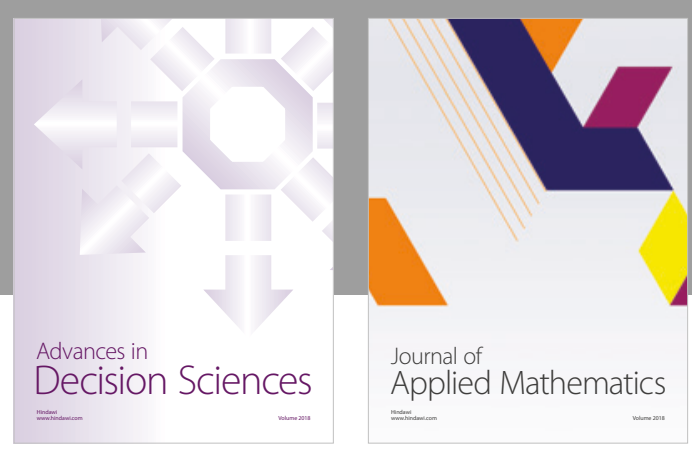

Journal of

Applied Mathematics
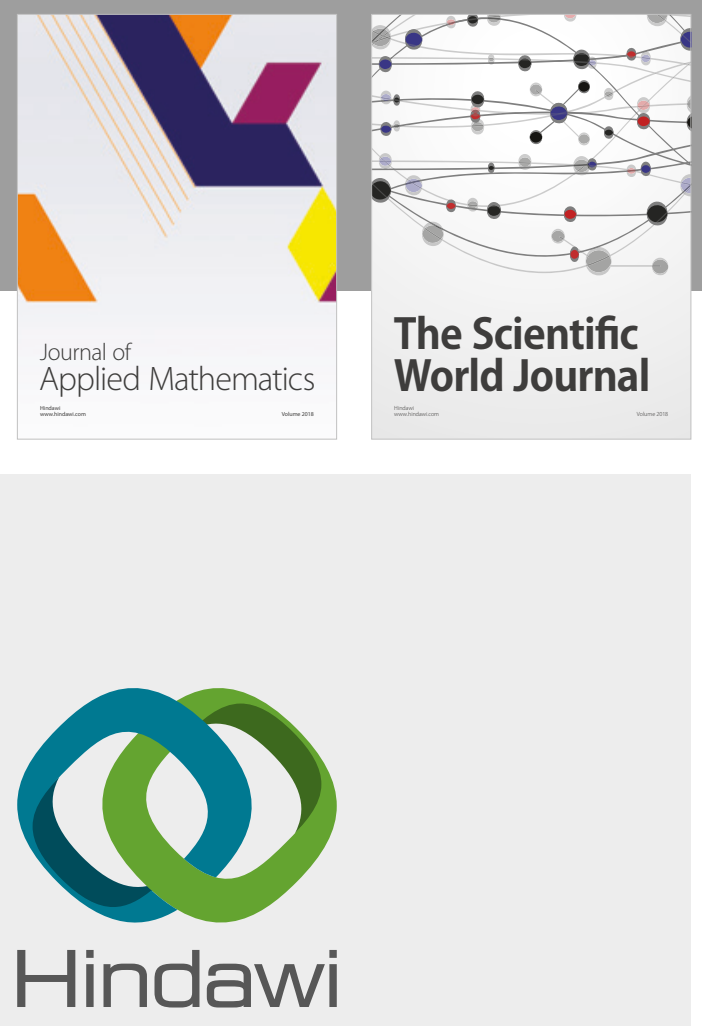

Submit your manuscripts at

www.hindawi.com

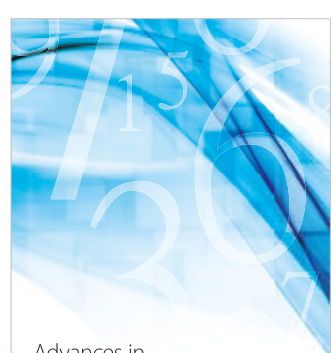

Advances in
Numerical Analysis
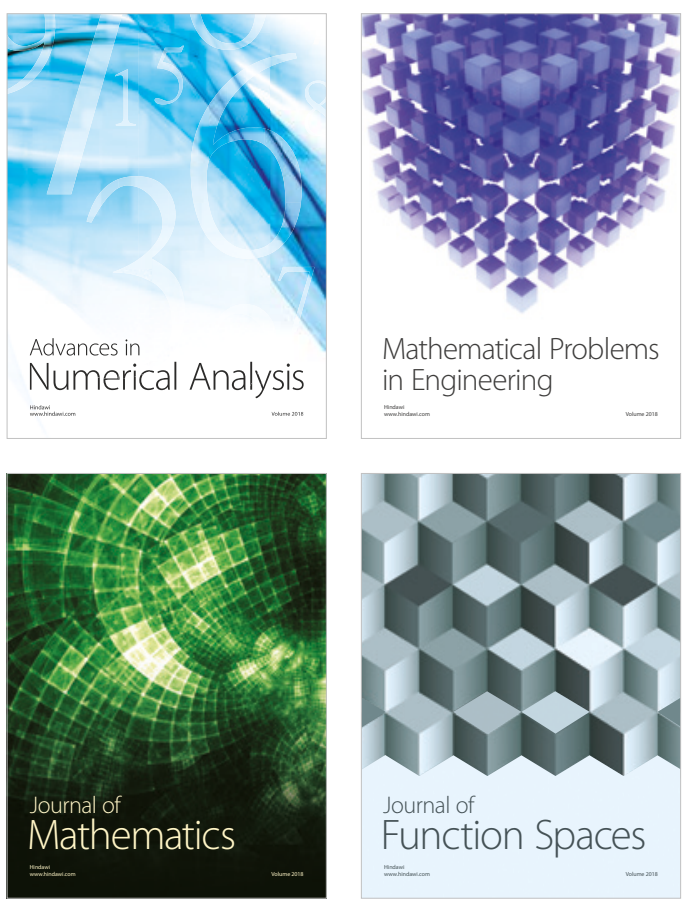

Mathematical Problems in Engineering

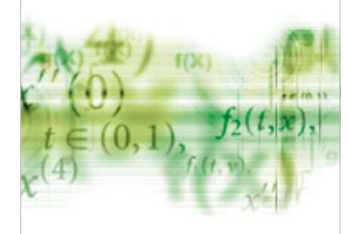

International Journal of

Differential Equations

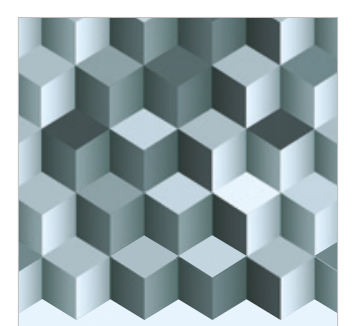

Journal of

Function Spaces

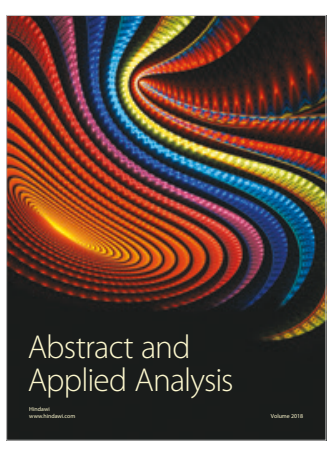

The Scientific

World Journal

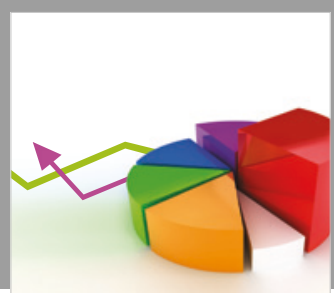

Journal of

Probability and Statistics
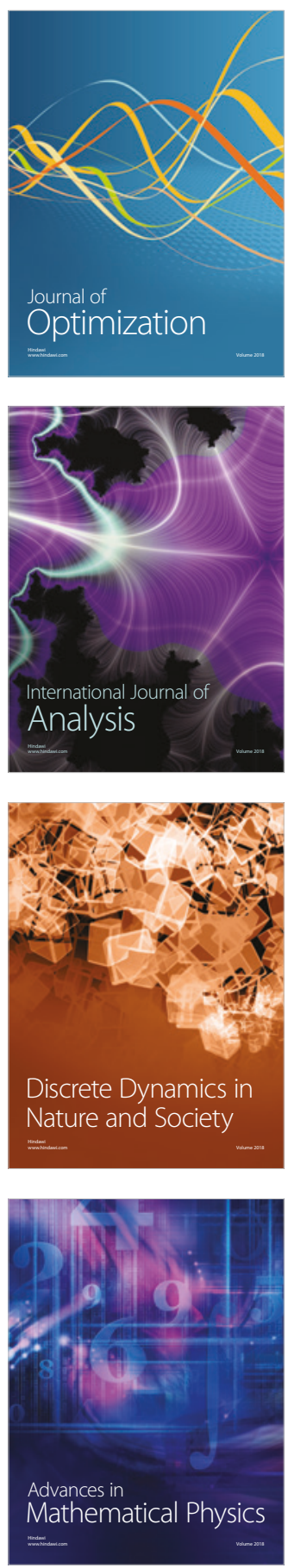